\title{
An optimal hydrodynamic model for the normal type IIP supernova $1999 \mathrm{em}$
}

\author{
V. P. Utrobin ${ }^{1,2}$ \\ 1 Max-Planck-Institut für Astrophysik, Karl-Schwarzschild-Str. 1, 85741 Garching, Germany \\ e-mail: utrobin@itep.ru \\ 2 Institute of Theoretical and Experimental Physics, B. Cheremushkinskaya St. 25, 117218 Moscow, Russia
}

Received 21 July 2006 / Accepted 22 August 2006

\begin{abstract}
Context. There is still no consensus about progenitor masses of type IIP supernovae.

Aims. We study a normal type IIP SN 1999em in detail and compare it to a peculiar type IIP SN 1987A.

Methods. We computed the hydrodynamic and time-dependent atmosphere models interpreting simultaneously both the photometric and spectroscopic observations.

Results. The bolometric light curve of SN 1999em and the spectral evolution of its $\mathrm{H} \alpha$ line are consistent with a presupernova radius of $500 \pm 200 R_{\odot}$, an ejecta mass of $19.0 \pm 1.2 M_{\odot}$, an explosion energy of $(1.3 \pm 0.1) \times 10^{51} \mathrm{erg}$, and a radioactive ${ }^{56} \mathrm{Ni}$ mass of $0.036 \pm 0.009 M_{\odot}$. A mutual mixing of hydrogen-rich and helium-rich matter in the inner layers of the ejecta guarantees a good fit of the calculated light curve to that observed. Based on the hydrodynamic models in the vicinity of the optimal model, we derive the approximate relationships between the basic physical and observed parameters. The hydrodynamic and atmosphere models of SN 1999em are inconsistent with the short distance of $7.85 \mathrm{Mpc}$ to the host galaxy.

Conclusions. We find that the hydrogen recombination in the atmosphere of a normal type IIP SN 1999em, as well as most likely other type IIP supernovae at the photospheric epoch, is essentially a time-dependent phenomenon. It is also shown that in normal type IIP supernovae the homologous expansion of the ejecta in its atmosphere takes place starting from nearly the third day after the supernova explosion. A comparison of SN 1999em with SN 1987A reveals two very important results for supernova theory. First, the comparability of the helium core masses and the explosion energies implies a unique explosion mechanism for these core collapse supernovae. Second, the optimal model for SN $1999 \mathrm{em}$ is characterized by a weaker ${ }^{56} \mathrm{Ni}$ mixing up to $\approx 660 \mathrm{~km} \mathrm{~s}^{-1}$ compared to a moderate ${ }^{56} \mathrm{Ni}$ mixing up to $\sim 3000 \mathrm{~km} \mathrm{~s}^{-1}$ in SN 1987A, hydrogen being mixed deeply downward to $\sim 650 \mathrm{~km} \mathrm{~s}^{-1}$.
\end{abstract}

Key words. stars: supernovae: individual: SN 1999em - stars: supernovae: individual: SN 1987A

\section{Introduction}

The supernova (SN) 1999em was discovered by the Lick Observatory Supernova Search on October 29.44 UT in the nearly face-on SBc galaxy NGC 1637 (Li 1999). Detected shortly after the explosion at an unfiltered magnitude of $\sim 13.5$, SN 1999em was bright enough to be observed well both photometrically and spectroscopically for more than 500 days (Hamuy et al. 2001; Leonard et al. 2002; Elmhamdi et al. 2003). SN 1999em was the first type II-plateau supernova (SN IIP) detected at both X-ray and radio wavelengths, being the least radio luminous and one of the least X-ray luminous SNe (Pooley et al. 2002). The X-ray data indicated the interaction between SN ejecta and a pre-SN wind with a low mass-loss rate of $\sim 2 \times$ $10^{-6} M_{\odot} \mathrm{yr}^{-1}$. Leonard et al. (2001) presented the first spectropolarimetry of SN IIP based on the optical observations of SN 1999em during 160 days after SN discovery. The weak continuum polarization increasing from $p \approx 0.2 \%$ on day 7 to $p \approx 0.5 \%$ in the final observations was detected with an unchanging polarization angle $\theta \approx 160^{\circ}$. To date this event has become the most studied normal SN IIP.

In order to study properly any individual object, an accurate distance is required in addition to high quality observations. It is this point that is not clear for SN $1999 \mathrm{em}$. There was a reasonable agreement in determining the distance to
SN 1999em using the expanding photosphere method (EPM; Kirshner \& Kwan 1974): $7.5 \pm 0.5 \mathrm{Mpc}$ (Hamuy et al. 2001), $8.2 \pm 0.6 \mathrm{Mpc}$ (Leonard et al. 2002), and 7.838 $\pm 0.331 \mathrm{Mpc}$ (Elmhamdi et al. 2003). However, Leonard et al. (2003) identified 41 Cepheid variable stars in the galaxy NGC 1637 and found that the Cepheid distance to the host galaxy was $11.7 \pm 1.0 \mathrm{Mpc}$. This distance to SN 1999em is $\sim 50 \%$ larger than the earlier EPM distance estimates. Assuming a correlation between the total ${ }^{56} \mathrm{Ni}$ mass and the explosion energy for SNe IIP, Nadyozhin (2003) evaluated the distance to the galaxy NGC 1637 of $11.08 \mathrm{Mpc}$ close to the Cepheid distance. Using the spectral-fitting expanding atmosphere model (SEAM), Baron et al. (2004) obtained the distance to SN 1999em of $12.5 \pm 1.8 \mathrm{Mpc}$ in a good agreement with the Cepheid distance scale. Finally, studying various ingredients entering the original EPM, Dessart \& Hillier (2006) improved this method and also achieved better agreement to the Cepheid distance with an estimate of $11.5 \pm 1.0 \mathrm{Mpc}$.

Starting the investigation of SN 1999em, a normal SN IIP, it is impossible to pass by the well-known and studied SN 1987A in the Large Magellanic Cloud (LMC), a peculiar SN IIP. Recent progress in reproducing the bolometric light curve observed in SN 1987A with a modern hydrodynamic model (Utrobin 2004) and in modelling the $\mathrm{H} \alpha$ profile and the Ba II $6142 \AA$ line in 
SN 1987A at the photospheric phase with a time-dependent approach for the SN atmosphere (Utrobin \& Chugai 2002, 2005) makes this experience very instructive for other SNe IIP. First, it turned out that a good agreement between the hydrodynamic models and the photometric observations of SN 1987A did not guarantee a correct description of this phenomenon as a whole. Second, the strength of the $\mathrm{H} \alpha$ line and its profile provided hard constraints on the hydrodynamic models. The most important lesson from this study of SN 1987A is that we have to take both the photometric and spectroscopic observations into account to obtain an adequate hydrodynamic model (Utrobin 2005). Now such an approach should be applied to SN 1999em.

Here we present the comprehensive hydrodynamic study of SN 1999em complemented by the atmosphere model with the time-dependent kinetics and energy balance. A brief description of the hydrodynamic model and the atmosphere model based on it is given in Sect. 2. The study of SN 1999em we begin with a construction of the optimal hydrodynamic model (Sect. 3). Then we continue with a question: at what distance to SN 1999em, short or long, the hydrodynamic model and the proper atmosphere model are consistent with the photometric and $\mathrm{H} \alpha$ line observations (Sect. 4). The time development of the optimal hydrodynamic model is presented in Sect. 5, and its general regularities in Sect. 6. The basic relationships between the physical and observed parameters for SNe IIP similar to the SN 1999em event are obtained in Sect. 7, while in Sect. 8 we address the comparison of SN 1999em with SN 1987A. In Sect. 9 we discuss our results from the theoretical and observational points of view. Finally in Sect. 10 we summarize the results obtained.

We adopt here a distance of 11.7 Mpc (Leonard et al. 2003), a recession velocity to SN $1999 \mathrm{em}$ of $800 \mathrm{~km} \mathrm{~s}^{-1}$ (Leonard et al. 2002), an explosion date of JD 2451476.77, and a total extinction $A_{\mathrm{V}}=0.31$ (Baron et al. 2000; Hamuy et al. 2001; Elmhamdi et al. 2003).

\section{Supernova modelling and input physics}

Keeping in mind the importance of a hydrodynamic study and of atmosphere modelling, including the time-dependent kinetics and energy balance for the interpretation of the SN 1987A phenomenon, we use this approach to investigate SN 1999em.

A hydrodynamic model is computed in terms of radiation hydrodynamics in the one-group approximation taking into account non-LTE effects on the average opacities and the thermal emissivity, effects of nonthermal ionization, and a contribution of lines to opacity as in the case of SN 1987A (Utrobin 2004). Note that the bolometric luminosity of an $\mathrm{SN}$ is calculated by including the retardation and limb-darkening effects.

The atmosphere model includes the time-dependent ionization and excitation kinetics of hydrogen and other elements, the time-dependent kinetics of molecular hydrogen, and the timedependent energy balance (Utrobin \& Chugai 2005). The density distribution, chemical composition, radius of the photosphere, and effective temperature are provided by the corresponding hydrodynamic model. The obtained time-dependent structure of the atmosphere is then used to calculate synthetic spectra at selected epochs. The spectra are modelled by the Monte Carlo technique suggesting that the photosphere diffusively reflects the incident photons and that the line scattering is generally non-conservative and is described in terms of the line scattering albedo. The Thomson scattering on free electrons and Rayleigh scattering on neutral hydrogen are also taken into account.
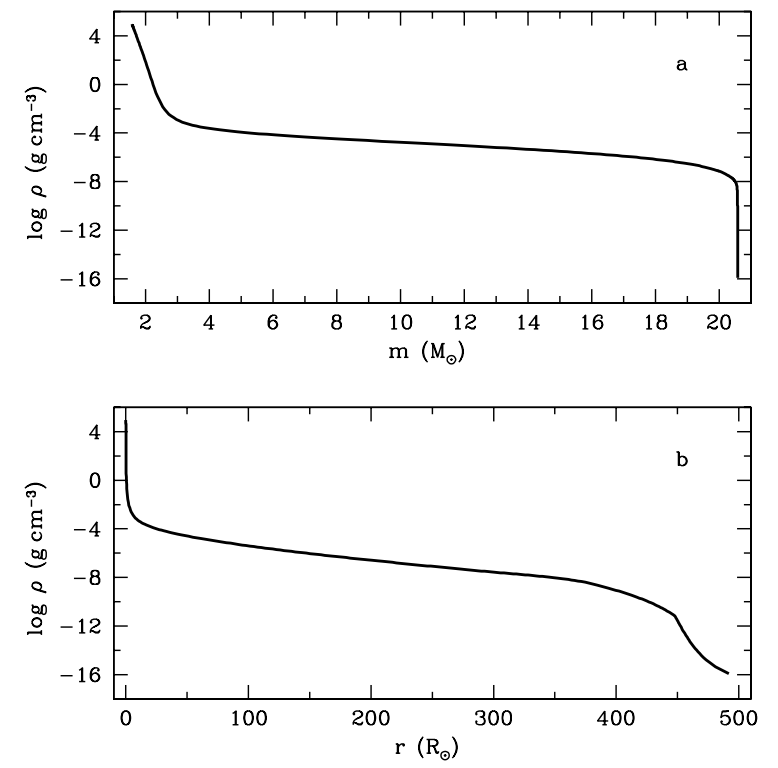

Fig. 1. Density distribution with respect to interior mass a) and radius b) for the pre-SN model D11. The central core of $1.58 M_{\odot}$ is omitted.

\section{Optimal hydrodynamic model}

Elmhamdi et al. (2003) have constructed the " $U B V R I$ " bolometric light curve of SN 1999em from the corresponding photometric data. To account for a possible contribution of the missing infrared bands, we add a value of 0.19 dex, taken from Elmhamdi et al. (2003), to the " $U B V R I$ " luminosity and adopt the resultant light curve as the bolometric light curve of SN 1999em. Our aim is to find an adequate hydrodynamic model that reproduces the photometric and spectroscopic observations of SN 1999em. To fit the bolometric light curve of SN $1999 \mathrm{em}$, various hydrodynamic models were explored. The bolometric light curve of SN 1999 em is fitted by adjusting the pre-SN radius $R_{0}$, the ejecta mass $M_{\mathrm{env}}$, and the explosion energy $E$, along with the density distribution in the pre-SN model and its chemical composition in the transition region between the hydrogen-rich envelope and metal/helium core. The radioactive ${ }^{56} \mathrm{Ni}$ mass is reliably measured by the light curve tail after the plateau phase. As the preSN model of SN 1999em, we consider non-evolutionary models similar to those of SN 1987A (Utrobin 2004), but for the outer layers assume the standard solar composition with the mass fraction of hydrogen $X=0.735$, helium $Y=0.248$, and the metallicity $Z=0.017$ (Grevesse \& Sauval 1998) taking a normal spiral nature of the host galaxy NGC 1637 into account. The best version of such a fitting was obtained with the optimal model that is characterized by the basic parameters: the pre-SN radius of $500 R_{\odot}$, the ejecta mass of $19 M_{\odot}$, and the explosion energy of $1.3 \times 10^{51} \mathrm{erg}$ (model D11 in Table 1$)$.

The density profile of the pre-SN model consisting of a central white dwarf like core and an outer envelope with the size of the red supergiant is shown in Fig. 1. In the calculations, the $1.58 M_{\odot}$ central core is removed from the computational mass grid and assumed to collapse to a neutron star, while the rest of the star is ejected by the SN explosion, which is modelled by a piston action near the edge of the central core. The pre-SN model has a heterogeneous chemical composition containing a $5.6 M_{\odot}$ helium core and a $11.9 M_{\odot}$ outer shell of the solar chemical composition (Fig. 2). Note that the helium cores up to about $8 M_{\odot}$ are consistent with the observations (Sect. 6.2). There is no sharp boundary between the 


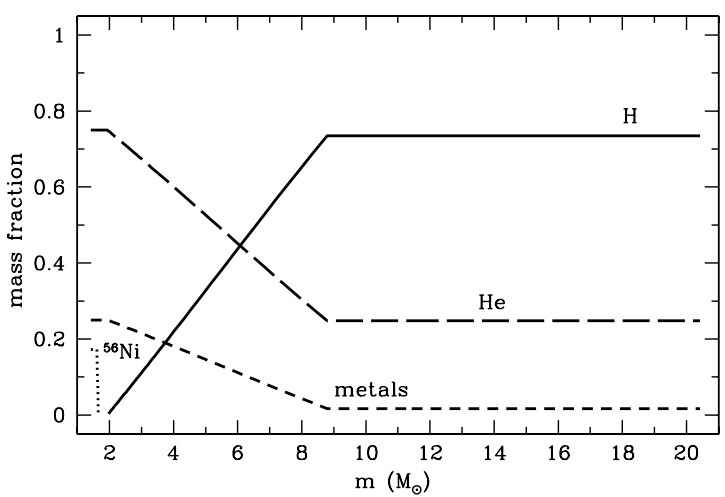

Fig. 2. The mass fraction of hydrogen (solid line), helium (long dashed line), heavy elements (short dashed line), and radioactive ${ }^{56} \mathrm{Ni}$ (dotted line) in the ejecta of model D11.

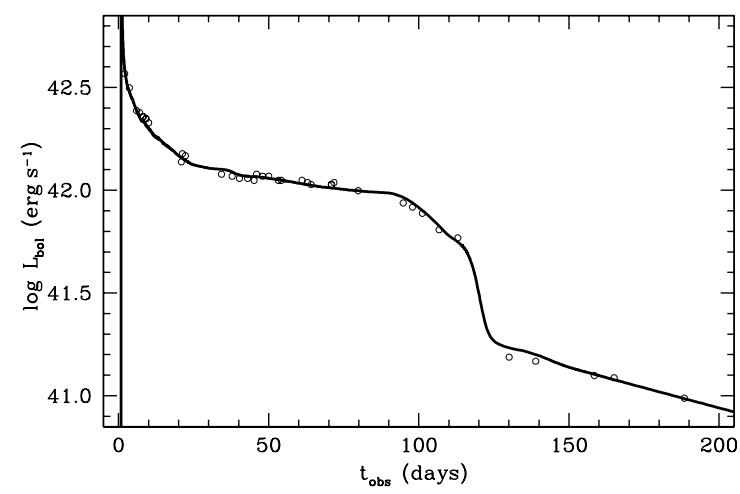

Fig. 3. Comparison of the calculated bolometric light curve of model D11 (solid line) with the bolometric data of SN 1999em obtained by Elmhamdi et al. (2003) (open circles).

hydrogen-rich and helium-rich layers in the ejecta of the optimal model. Hydrogen-rich material is mixed into the central region, and helium-rich material, in turn, is mixed outwards. It is evident that such a distribution of hydrogen and helium implies a strong mixing at the helium/hydrogen composition interface. The fact that the radioactive ${ }^{56} \mathrm{Ni}$ is confined to the innermost ejected layers suggests its weak mixing during the SN explosion.

In Fig. 3 we show the very good match between the bolometric light curve calculated for the optimal hydrodynamic model and the one observed for SN 1999em (Elmhamdi et al. 2003). Note that hereafter $t_{\mathrm{obs}}$ is the time in the observer's frame of reference. The model agrees well with the observed tail of the bolometric light curve for the total ${ }^{56} \mathrm{Ni}$ mass of $0.036 M_{\odot}$, the bulk of the radioactive ${ }^{56} \mathrm{Ni}$ being mixed in the velocity range $\leq 660 \mathrm{~km} \mathrm{~s}^{-1}$ (Fig. 10) in order to reproduce the observed transition from the plateau to the tail.

\section{H $\alpha$ profile: evidence against short distance}

It is quite clear that any well-observed SN should be described by a unique hydrodynamic model in combination with the atmosphere model based on it. The experience during the study of SN 1987A showed that such a combination of the models had to fit not only the photometric, but also the spectroscopic observations. We believe that the adequate hydrodynamic and atmosphere models of SN 1999em are able to distinguish between the short and long distances. A distance of $7.85 \mathrm{Mpc}$, the average value of the EPM distance estimates, is taken as the short
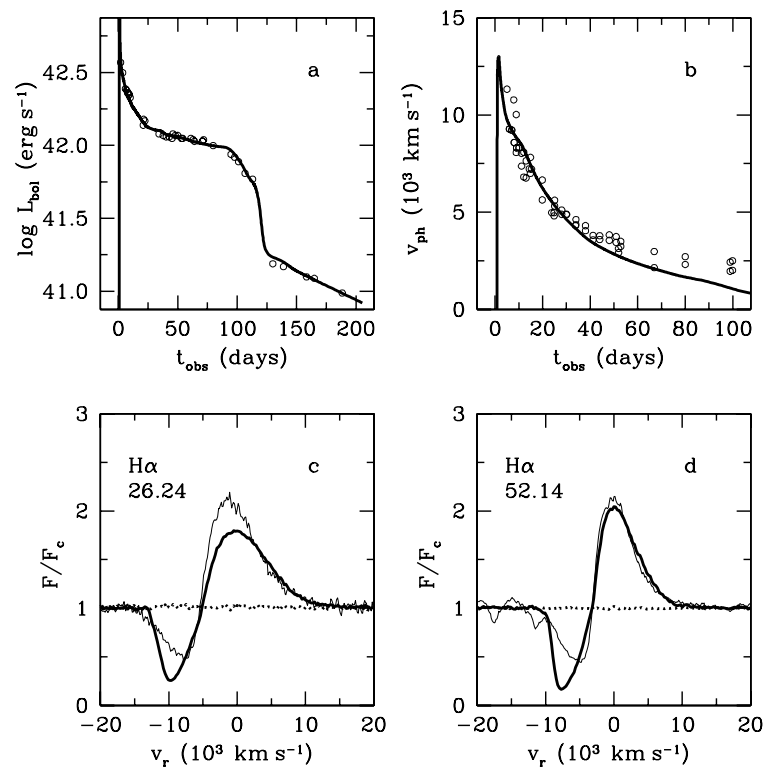

Fig. 4. Hydrodynamic model D11 and $\mathrm{H} \alpha$ line for the distance of $11.7 \mathrm{Mpc}$. Panel a): the calculated bolometric light curve (solid line) compared with the observations of SN 1999em obtained by Elmhamdi et al. (2003) (open circles). Panel b): calculated photospheric velocity (solid line) and radial velocities at maximum absorption of spectral lines measured by Hamuy et al. (2001) and Leonard et al. (2002) (open circles). Panel c): $\mathrm{H} \alpha$ profiles, computed with the time-dependent approach (thick solid line) and with the steady-state model (dotted line), overplotted on the observed profile on day 26.24, as obtained by Leonard et al. (2002) (thin solid line). Panel d): the same as panel c) but for day 52.14 .

distance, and the Cepheid distance of $11.7 \mathrm{Mpc}$ is taken as the long distance.

As demonstrated above, we constructed the optimal hydrodynamic model for the long distance (model D11 in Table 1). The model reproduces the observed bolometric light curve of SN 1999em very well (Fig. 4a). In Fig. 4b the calculated expansion velocity at the photosphere level, the photospheric velocity, is compared with the radial velocities at maximum absorption of the different spectral lines measured by Hamuy et al. (2001) and Leonard et al. (2002). The photospheric velocity of model D11 is consistent with the observed points, at least for the first 60 days. To verify the hydrodynamic model by matching the constraints from the spectral observations of SN 1999em, we examined the $\mathrm{H} \alpha$ profile on days 26.24 and 52.14 computed in the time-dependent approach.

In the time-dependent atmosphere model for SN 1987A, we considered two extreme cases to allow for the uncertainty of our approximation in a description of the ultraviolet radiation field: (i) the photospheric brightness is black-body with the effective temperature (model A); (ii) the photospheric brightness corresponds to the observed spectrum (model B) (Utrobin \& Chugai 2005). For SN 1999em the photospheric brightness in model B is black-body with the effective temperature and the corresponding brightness reduction taken from the SN 1987A observations.

The time-dependent approach with model B satisfactorily reproduces the strength of the $\mathrm{H} \alpha$ emission component on day 26.24 with some emission deficit near the maximum in comparison to what was observed (Fig. 4c). This deficit is not significant because model A, calculated for this phase and not plotted on Fig. 4c for the sake of clarity, gives the emission component with a relative flux of 3.1 at the maximum, which is 
Table 1. Hydrodynamic models for distances of 7.85 and $11.7 \mathrm{Mpc}$.

\begin{tabular}{ccccccc}
\hline \hline Model & $\begin{array}{c}R_{0} \\
\left(R_{\odot}\right)\end{array}$ & $\begin{array}{c}M_{\text {env }} \\
\left(M_{\odot}\right)\end{array}$ & $\begin{array}{c}E \\
\left(10^{51} \mathrm{erg}\right)\end{array}$ & $\begin{array}{c}M_{\mathrm{Ni}} \\
\left(10^{-2} M_{\odot}\right)\end{array}$ & $X$ & $Z$ \\
\hline D07 & 375 & 16 & 0.686 & 1.62 & 0.735 & 0.017 \\
D11 & 500 & 19 & 1.300 & 3.60 & 0.735 & 0.017 \\
\hline
\end{tabular}
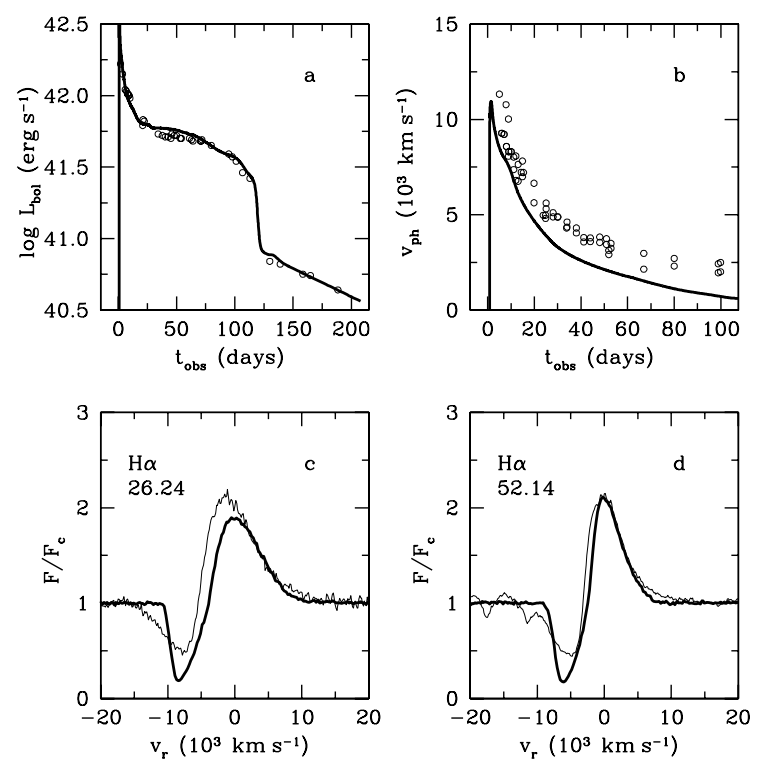

Fig. 5. Hydrodynamic model D07 and $\mathrm{H} \alpha$ line for the distance of $7.85 \mathrm{Mpc}$. See the Fig. 4 legend for details.

much higher than the observed one, so the real situation is somewhere between these two cases and is closer to model B. Note that in SN 1987A the $\mathrm{H} \alpha$ emission component demonstrated the same behavior in the early phase (Utrobin \& Chugai 2005). In contrast, the $\mathrm{H} \alpha$ absorption component calculated in model B is stronger than that observed on day 26.24 in SN 1999em. This discrepancy is presumably related to a poor description of the ultraviolet radiation at frequencies between the Balmer and Lyman edges. This radiation interacts with numerous metal lines and controls the populations of hydrogen levels. It is very important that both the emission and absorption components of the $\mathrm{H} \alpha$ line calculated in model B extend over the whole range of the observed radial velocities from $-15000 \mathrm{~km} \mathrm{~s}^{-1}$ to $15000 \mathrm{~km} \mathrm{~s}^{-1}$ (Fig. 4c). Unfortunately, the calculated absorption component runs above the observed one in a radial velocity range between $-15000 \mathrm{~km} \mathrm{~s}^{-1}$ and $-12500 \mathrm{~km} \mathrm{~s}^{-1}$. On day 52.14 the emission component of the $\mathrm{H} \alpha$ line computed with the time-dependent approach with model B fits the observed one fairly well, while the absorption component is still stronger than the observed one (Fig. 4d). Thus, we may state that the above hydrodynamic and atmosphere models are in a good agreement with the photometric and spectroscopic observations of SN 1999em.

Now let us pay attention to the time-dependent effects in hydrogen lines of SN 1999em, a normal SN IIP. The timedependent approach with model $\mathrm{B}$ reproduces the strength of the $\mathrm{H} \alpha$ line at day 26.24 and day 52.14 fairly well (Figs. 4c and d). In contrast, a steady-state model B demonstrates an extremely weak $\mathrm{H} \alpha$ line on days 26.24 and 52.14. This reflects the fact that the steady-state ionization is significantly lower than in the time-dependent model, while the electron temperature is too low for the collisional excitation of hydrogen. The radioactive ${ }^{56} \mathrm{Ni}$ is mixed too weakly to affect the ionization and excitation of hydrogen and other elements in the atmosphere at the plateau

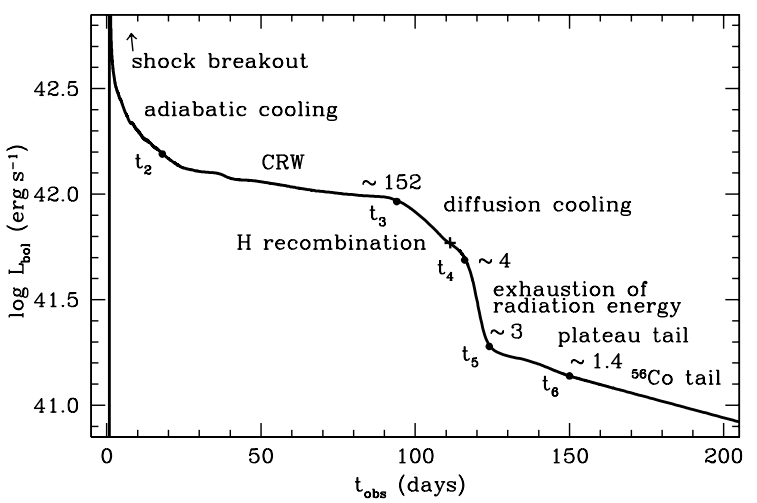

Fig. 6. Evolution of a normal SN IIP illustrated by the bolometric light curve of the optimal model. Lettered dots mark the specific times in the evolution. The end time of the shock breakout phase, $t_{1}$, is shown in Fig. 8a. The cross notes the moment of complete hydrogen recombination. The numbers indicate the total optical depth of the ejecta at the corresponding times.

phase. Thus, it is possible to conclude that the hydrogen recombination in the atmosphere of SN 1999em during the whole plateau phase is essentially a time-dependent phenomenon.

In turn, the short distance results in the hydrodynamic model with the following parameters: the pre-SN radius of $375 R_{\odot}$, the ejecta mass of $16 M_{\odot}$, and the explosion energy of $6.86 \times$ $10^{50}$ erg (model D07 in Table 1). In this model the central core of $1.4 M_{\odot}$ is assumed to collapse to a neutron star. The hydrodynamic model fits the observed bolometric light curve of SN $1999 \mathrm{em}$ but for the ${ }^{56} \mathrm{Ni}$ mass of $0.0162 M_{\odot}$, the most of ${ }^{56} \mathrm{Ni}$ being mixed in the velocity range $\leq 580 \mathrm{~km} \mathrm{~s}^{-1}$ (Fig. 5a). The photospheric velocity curve runs well below the observed points (Fig. 5b), and it might be expected that the spectral lines in this model would be narrower than those observed. Indeed, on days 26.24 and 52.14 both the emission and absorption components of the $\mathrm{H} \alpha$ line computed with the time-dependent approach with model B are significantly narrower than those observed in SN 1999em (Figs. 5c and 5d). The hydrodynamic model D07 agrees fairly well with the observed bolometric light curve, but the relevant atmosphere model fails to reproduce the $\mathrm{H} \alpha$ profile observed in SN 1999em. We thus conclude that the short distance of $7.85 \mathrm{Mpc}$ should be discarded.

\section{Development of the optimal model}

Although the major issues of light curve theory were recognized a long time ago (Grassberg et al. 1971; Falk \& Arnett 1977), it is useful to consider an SN outburst in a more detailed approach. This study of SN 1999em provides a good opportunity to examine the time development of a normal SN IIP. Figures 6 and 8a show the following stages in the observer time scale: a shock breakout $\left(t_{\mathrm{obs}} \leq t_{1}\right)$, an adiabatic cooling phase $\left(t_{1}<t_{\mathrm{obs}} \leq t_{2}\right)$, a phase of cooling and recombination wave (CRW) $\left(t_{2}<t_{\mathrm{obs}} \leq t_{3}\right)$, a phase of radiative diffusion cooling $\left(t_{3}<t_{\mathrm{obs}} \leq t_{4}\right)$, an exhaustion of radiation energy $\left(t_{4}<t_{\mathrm{obs}} \leq t_{5}\right)$, a plateau tail $\left(t_{5}<t_{\mathrm{obs}} \leq t_{6}\right)$, and a radioactive tail $\left(t_{\mathrm{obs}}>t_{6}\right)$. In addition to the above stages there are two specific points: a transition from acceleration of the envelope matter to a homologous expansion and a moment of complete hydrogen recombination. In the optimal model D11, the characteristic moments are $t_{1} \approx 0.93$ days, $t_{2} \approx 18$ days, $t_{3} \approx 94$ days, $t_{4} \approx 116$ days, $t_{5} \approx 124$ days, and $t_{6} \approx 150$ days, and the complete hydrogen 


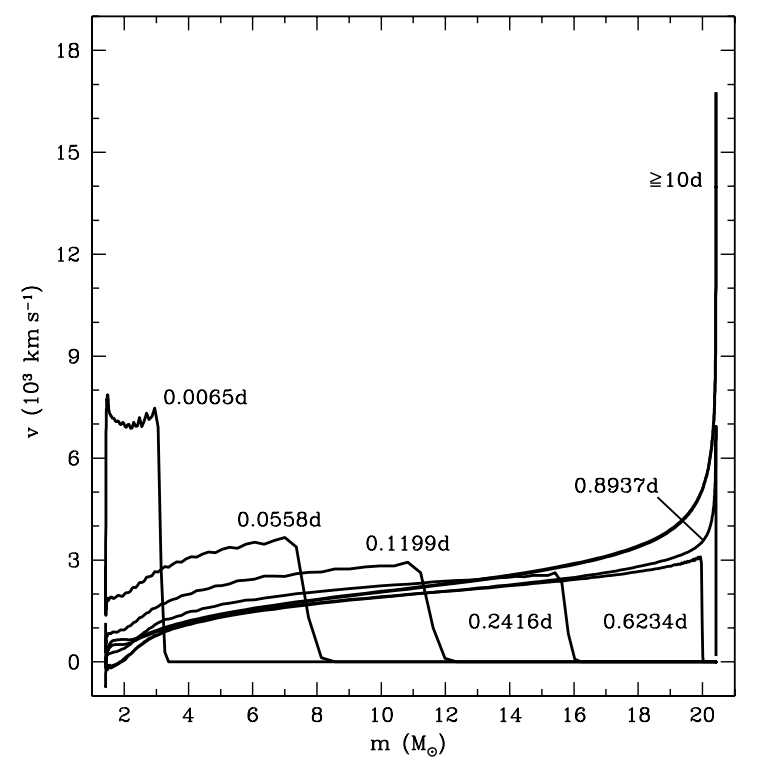

Fig. 7. Propagation of the shock through the ejected envelope of model D11. Velocity profiles with respect to interior mass are plotted at $t=0.0065,0.0558,0.1199,0.2416,0.6234,0.8937$ days, and $t \geq 10$ days after the $\mathrm{SN}$ explosion.

recombination occurs at $t_{\mathrm{H}} \approx 111.3$ days. Below we consider the basic stages in the time development of the optimal model.

\subsection{Shock breakout}

The explosion of the star is assumed to be triggered by a piston action near the edge of the central core immediately after the epoch zero, $t=0$. From here on, $t$ is the time in the comoving frame of reference. This energy release generates a strong shock wave that propagates towards the stellar surface. In moving out of the center, the shock wave heats matter and accelerates it to velocities increasing outward and exceeding the local escape velocity. From $t=0.0065$ days to $t=0.2416$ days, the shock wave, propagating outward from the compact dense core of the pre-SN (Fig. 1), is attenuated slightly due to the spherical divergence (Fig. 7). It then reaches the outermost layers with a sharp decline in density and after $t=0.6234$ days gains strength and accelerates due to the effect of hydrodynamic cumulation. Only a small portion of the star undergoes this acceleration and acquires a high velocity. For example, the layers of velocities exceeding $5000 \mathrm{~km} \mathrm{~s}^{-1}$ have a mass of $\approx 0.478 M_{\odot}$.

By day 0.8651 the shock wave reaches the stellar surface and then begins to heat the external layers, so that the color temperature jumps to $3.84 \times 10^{5} \mathrm{~K}$ at day 0.8823 , the effective temperature increases up to $1.76 \times 10^{5} \mathrm{~K}$ at day 0.8943 , and the bolometric luminosity rises accordingly up to $6.46 \times 10^{44} \mathrm{erg} \mathrm{s}^{-1}$ at day 0.8943 (Fig. 8). Note that the maximum of the color temperature coincides closely with the moment of 0.8803 days when a velocity at the stellar surface reaches the escape velocity.

A very rapid rise in the bolometric luminosity to maximum, starting at day 0.8651 , instantly changes a growth of the photospheric radius into its reduction because of the intense radiative losses of energy in the outermost layers (Fig. 8b). At day 0.8691 these layers begin to move outward, and the additional cooling by adiabatic expansion makes the reduction of the photospheric radius more noticeable. At the same time the envelope expansion is favorable to a photon diffusion, decreasing the characteristic diffusion time. The photon diffusion eventually dominates, stops
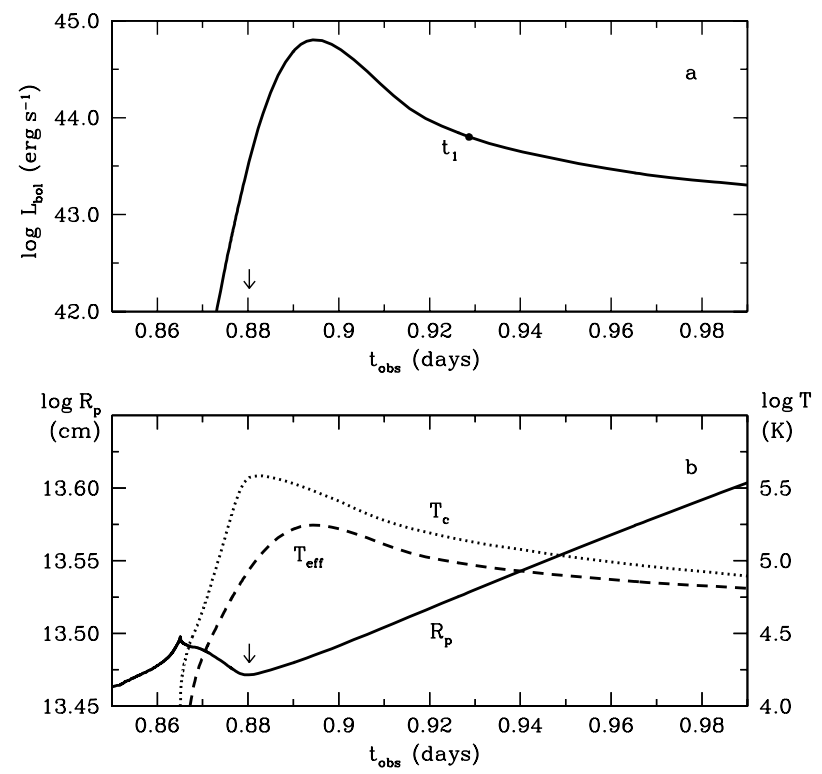

Fig. 8. Shock breakout in model D11. Arrow indicates the moment of 0.8803 days when the velocity at the stellar surface reaches escape velocity. Panel a): the calculated bolometric light curve and the lettered dot marking the end time of the shock breakout phase. Panel b): the calculated photospheric radius (solid line), the effective temperature (dashed line), and the color temperature (dotted line) as a function of the observer's time.

this reduction at day 0.8802 , and then, along with the envelope expansion, blows the photospheric radius outward.

When a velocity at the stellar surface exceeds the escape velocity, the outside layers of the star begin to cool rapidly, the color temperature begins to decrease from its maximum value, and both the effective temperature and the luminosity decrease somewhat later. A narrow peak in the bolometric luminosity forms as a result (Fig. 8a). The peak has a width of about 0.02 days at a half level of the luminosity maximum. Most of its radiation is emitted in an ultraviolet flash. The total number of ionizing photons above $13.598 \mathrm{eV}$ for the whole outburst is $2.768 \times 10^{58}$. During the first 1.234 days the number of ionizing photons is $90 \%$ of the total number, and the radiated energy adds up to $1.59 \times 10^{48} \mathrm{erg}$. We conditionally define the transition time between the shock breakout and the adiabatic cooling phase, $t_{1}$, as the time at which the bolometric luminosity drops by one dex from its maximum value, with the understanding that the adiabatic cooling becomes essential soon after the onset of the envelope expansion. In the optimal model this transition time is nearly 0.93 days.

Scattering processes are fundamentally nonlocal and result in exceeding the color temperature over the effective one when they are dominant (Mihalas 1978; Sobolev 1980). During the shock breakout, the adiabatic cooling phase and the CRW phase scattering processes dominate those of true absorption near the photospheric level in the ejecta of the optimal model and, as a consequence, lead to a color temperature that is considerably higher than the effective temperature. Figure $8 \mathrm{~b}$ shows that the maximum of the color temperature coincides very closely in time with the local minimum of the photospheric radius at day 0.8802 and comes before the maximum of the effective temperature and the bolometric luminosity. 


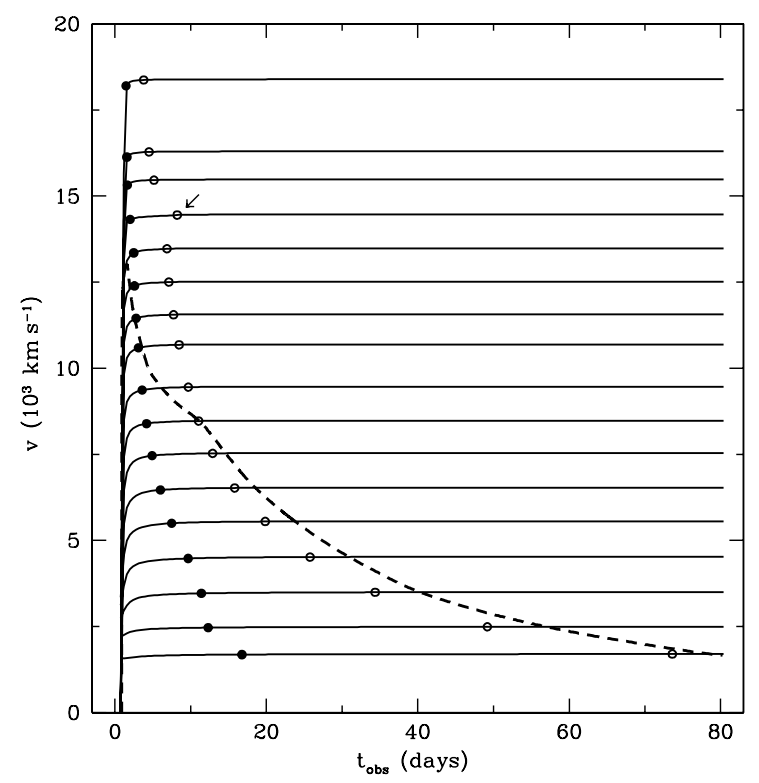

Fig. 9. Time dependence of the velocity of different mass shells (solid line) in the ejected envelope of model D11 and the photospheric velocity (dashed line). Full and open circles indicate moments when a velocity of the mass shell reaches $99 \%$ and $99.9 \%$, respectively, of its terminal velocity. The arrow marks the mass shell that has undergone an additional acceleration due to the expansion opacity.

\subsection{Adiabatic cooling phase}

During the shock passage throughout the star, the gas is heated up to about $10^{5} \mathrm{~K}$ and, as a consequence, is totally ionized. Both the internal gas energy and the radiation energy increase in the SN envelope. After the shock breakout, a dominant process in the subphotospheric, optically thick layers of the expelled envelope is the cooling by adiabatic expansion. The adiabatic losses drastically reduce the stored energy and completely determine the evolution of the bolometric luminosity during the adiabatic cooling phase (Figs. 6 and 8a). Such behavior of the luminosity lasts till the gas and radiation temperatures drop to the critical value in the subphotospheric layers, and a recombination of hydrogen, the most abundant element in the ejected envelope, starts. In these layers hydrogen becomes partially ionized by around day 18 . For the adiabatic cooling phase, the color and effective temperatures drop rapidly from $1.35 \times 10^{5} \mathrm{~K}$ and $9.32 \times 10^{4} \mathrm{~K}$, respectively, at day 0.93 to $6650 \mathrm{~K}$ and $6560 \mathrm{~K}$ at day 18 . Their ratio also reduces as the contribution of scattering processes to the opacity decreases.

In an optically thick medium, the strong shock wave propagates almost adiabatically throughout the stellar matter. When the shock wave emerges on the pre-SN surface, the adiabatic regime is broken and transforms into the isothermal regime. This transformation takes place during the adiabatic cooling phase and gives rise to a thin, dense shell at the outer edge of the ejected envelope. The dense shell is arising from day 0.945 to day 1.022 , reaching the density contrast of $\sim 210$ at a velocity of $\sim 12300 \mathrm{~km} \mathrm{c}^{-1}$. The formation of the dense shell starts in the optically thick medium at the optical depth of $\sim 8$, and ends in semi-transparent medium at the optical depth of $\sim 1$. By day 18 the shell accelerates to a velocity of $\sim 13400 \mathrm{~km} \mathrm{c}^{-1}$ reducing the density contrast to a value of $\sim 2.5$. The matter in the dense shell is subject to the Rayleigh-Taylor instability, which may result in the strong mixing of matter (Falk \& Arnett 1977). The latter, in

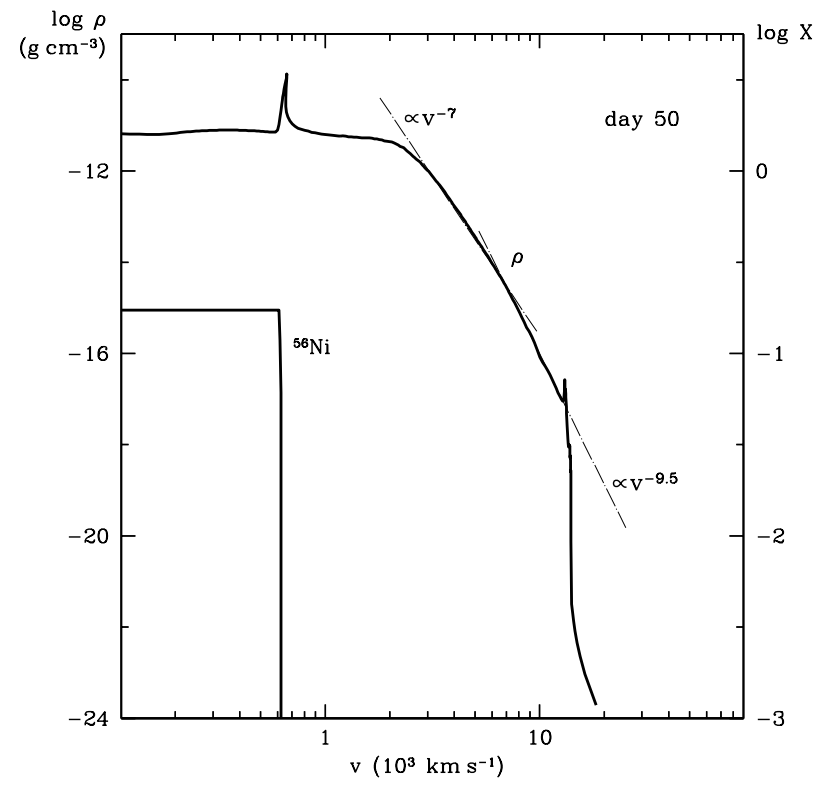

Fig. 10. The density and the ${ }^{56} \mathrm{Ni}$ mass fraction as a function of the velocity for model D11 at $t=50$ days. Dot-dash lines are the density distribution fits $\rho \propto v^{-7}$ and $\rho \propto v^{-9.5}$.

turn, may prevent the thin shell-like structure from forming in the outer layers of the envelope.

\subsection{Homologous expansion}

The SN explosion causes an acceleration of the envelope matter. At late times, when the acceleration of the ejecta becomes negligible, the envelope matter expands homologously. Evidently, there is a transition from the acceleration to the homologous expansion. This transition for different mass shells occurs at different times: the deeper the layer, the later the transition (Fig. 9). Thus, the transition time of the whole expelled envelope is determined by the deepest layer. If we consider physical processes in an $\mathrm{SN}$ atmosphere, the relevant transition time is given by the photospheric location. It is clear that the transition time for the SN atmosphere is much shorter than for the whole envelope. For instance, the atmosphere layers exceed $99 \%$ and $99.9 \%$ of their terminal velocities starting from nearly day 2.8 and day 11 , respectively (Fig. 9).

It is interesting that a monotonic increase in the transition time with embedding inward the ejecta is broken at the 99.9\% level of the terminal velocity. There is a prominent feature at a velocity of $\sim 14500 \mathrm{~km} \mathrm{c}^{-1}$ marked in Fig. 9. As we show later in Sect. 6.5, it is the result of an additional acceleration due to the resonance scattering in numerous metal lines.

Homologous expansion is characterized by a density distribution frozen in velocity space and scaled in time as $\propto t^{-3}$. Such a density profile for the optimal model is shown at $t=50$ days in Fig. 10. In the inner region of the ejected envelope there is a dense shell with a density contrast of $\sim 20$ produced by the ${ }^{56} \mathrm{Ni}$ bubble at a velocity of $\sim 660 \mathrm{~km} \mathrm{c}^{-1}$. The density distribution above the ${ }^{56} \mathrm{Ni}$ bubble shell is nearly uniform up to $\sim 2000 \mathrm{~km} \mathrm{c}^{-1}$. The outer layers with velocities in the ranges $3000-6900 \mathrm{~km} \mathrm{~s}^{-1}$ and $6900-13000 \mathrm{~km} \mathrm{~s}^{-1}$ may be fitted by an effective index $n=-\partial \ln \rho / \partial \ln r$ of 7 and 9.5 , respectively, as seen from the density distribution with respect to the expansion velocity (Fig. 10). At a velocity of $\sim 13000 \mathrm{~km} \mathrm{c}^{-1}$, there is another dense shell with a density contrast of $\sim 3$ originated during 

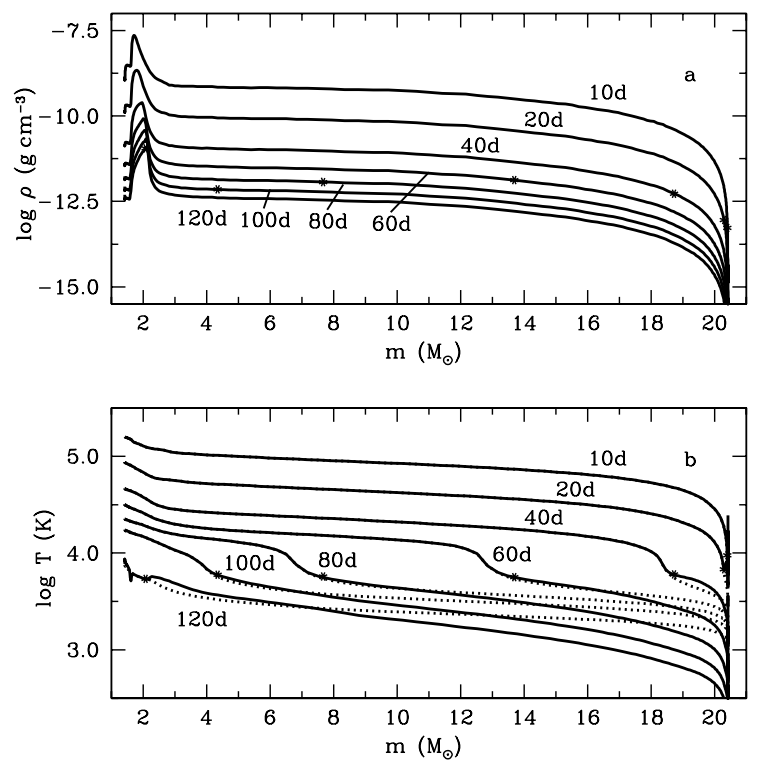

Fig. 11. Evolution of the density a) and the gas (solid line) and radiation (dotted line) temperatures $\mathbf{b}$ ) as a function of mass for model D11. Profiles are plotted at $t=10,20,40,60,80,100$, and 120 days. Star indicates the position of the photosphere.

the adiabatic cooling phase. Note that both dense shells slightly change their density profiles with time. For example, the evolution of the ${ }^{56} \mathrm{Ni}$ bubble shell is clearly demonstrated by Fig. 11a.

\subsection{Cooling and recombination wave}

As the envelope expands, cooling by radiation - a cooling and recombination wave - occurs and completely dominates the luminosity of the $\mathrm{SN}$ by about day 18 . From this time to nearly day 94 the bolometric luminosity is mainly determined by properties of the CRW (for details see Grassberg \& Nadyozhin 1976; Imshennik \& Nadyozhin 1989). A main property of the CRW consists in generating virtually the entire energy flux carried away by radiation within its front. This radiation flux exhausts the thermal and recombination energy of cooling matter by means of recombination. At the inner edge of the CRW, the radiation flux is negligibly small and matter is ionized, but at the outer edge the flux is equal to the luminosity of the star, and matter completely recombines.

During the CRW phase the evolution of the density in the ejecta illustrates the homologous expansion (Fig. 11a). The behavior of the gas and radiation temperatures reflects two important facts (Fig. 11b). First, in the subphotospheric layers in an optically thick medium, under conditions very close to LTE, the radiation temperature is virtually equal to the gas temperature. Second, in the transparent layers, beyond the photosphere, the gas temperature turns out to be lower than the radiation temperature because of the weak interaction between matter and radiation and of the dominant role of adiabatic losses. From an inspection of the interior luminosity and the fraction of ionized hydrogen from $t=20$ days to $t=100$ days, the CRW characteristics described above are evident (Figs. 12a and 12b). Hence in this period the CRW occurs.

From $t=20$ days to $t=100$ days, the CRW propagates through a mass of $\approx 15.96 M_{\odot}$, while the outermost layers, those responsible for the luminosity during the first 18 days, contribute only about $0.13 M_{\odot}$. It is interesting that for this period the gas and radiation temperatures at the photosphere position are nearly
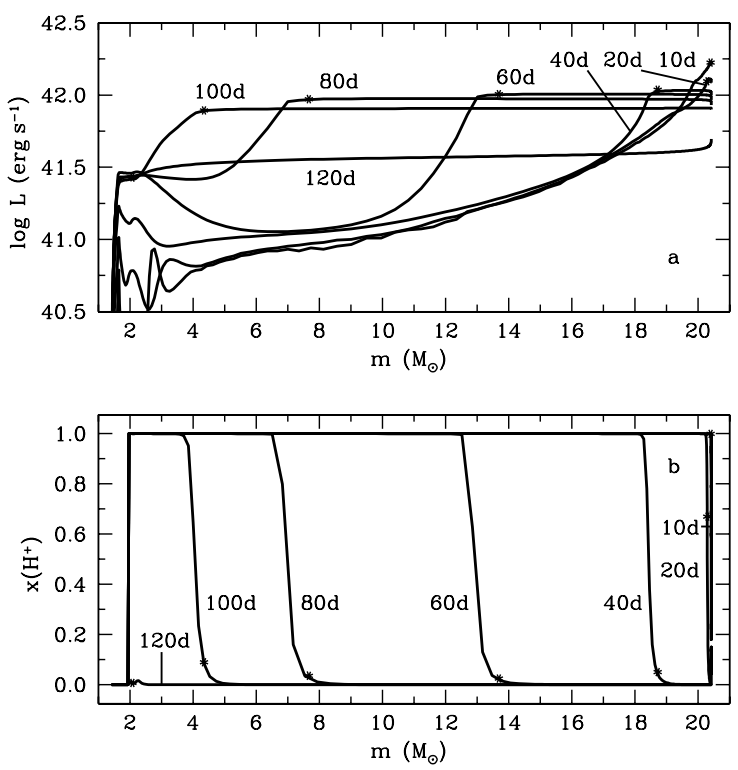

Fig. 12. Evolution of the interior luminosity a) and the fraction of ionized hydrogen b) as a function of mass for model D11. Profiles are plotted at $t=10,20,40,60,80,100$, and 120 days. Star indicates the position of the photosphere.

constant in time and equal to about $5600 \mathrm{~K}$ (Fig. 11b). And from $t=40$ days to $t=100$ days, the photosphere is located at the outer edge of the hydrogen recombination front (Fig. 12b).

After $t=100$ days the radiation flux at the inner edge of the CRW becomes comparable to the luminosity of star (Fig. 12a). This effect is caused by radiative diffusion from the central envelope layers where the energy of radioactive decays is being released. Later on the luminosity is entirely determined by radiative diffusion and not by the CRW.

\subsection{Radiative diffusion cooling}

A cooling by radiative diffusion starts in the optically thick expelled envelope at day 94 and ends in the semi-transparent medium at day 116 (Fig. 6). Shortly before the end of the phase of radiative diffusion cooling, the complete hydrogen recombination occurs at $t_{\mathrm{H}} \approx 111.3$ days. In the optimal model the radiative diffusion takes place in the inner layers with the nearly uniform density distribution and results in the characteristic shoulder of the bolometric luminosity. It should be noted that this behavior of the light curve was discussed by Falk \& Arnett (1977). To describe photon diffusion, we may use a simple onezone approximation (Arnett 1979), ignoring the contribution of radioactive decays to the bolometric light curve:

$\frac{\mathrm{d} \ln L_{\mathrm{bol}}}{\mathrm{d} t}=-\frac{1}{t_{\mathrm{dif}}}, t_{\mathrm{dif}}=\frac{\pi}{3} \frac{R_{\mathrm{d}}}{c} \tau_{\mathrm{d}}$

where $t_{\mathrm{dif}}$ is the diffusion time, $R_{\mathrm{d}}$ the characteristic radius of the diffusion region, and $\tau_{\mathrm{d}}$ the optical depth of this region.

Evidently, it is possible to estimate the diffusion time from both the decline of the bolometric light curve and the physical properties of the diffusion region. The decline of the luminosity shoulder between day 94 and day 116 measures a diffusion time of $\approx 35$ days (Fig. 6). On the other hand, the radius of the diffusion region of $\approx 1.2 \times 10^{15} \mathrm{~cm}$ and its optical depth of $\approx 63$ evaluated at day 105 give a diffusion time of $\approx 30$ days. These estimates are consistent with the above description of photon diffusion (1). Thus, the luminosity shoulder at the phase of 

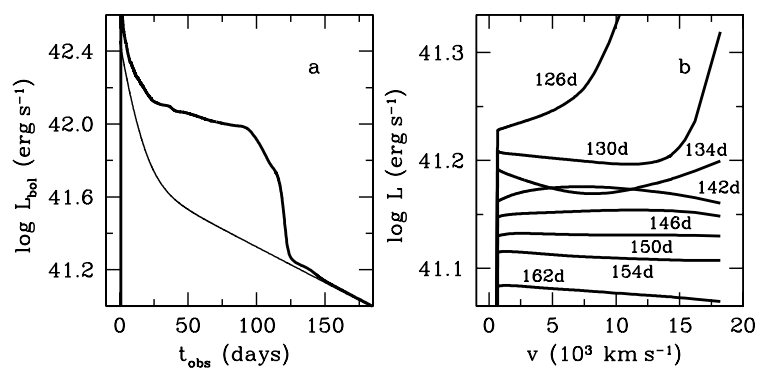

Fig. 13. The plateau tail of the bolometric light curve in the optimal model. Panel a): calculated bolometric light curve (thick solid line) is compared with the gamma-ray luminosity (thin solid line). Panel b): evolution of the interior luminosity as a function of velocity. Profiles are plotted at $t=126,130,134,142,146,150,154$, and 162 days.

radiative diffusion cooling is approximated well by the simple model with the constant diffusion time. Note that a transition from the constant diffusion time to the variable diffusion time, which is getting shorter, transforms the linear shoulder of the luminosity in logarithmic scale into a convex light curve at the end of the plateau.

\subsection{Exhaustion of radiation energy}

During the entire outburst, the total radiation energy in the ejecta is much greater than its total internal gas energy. In the inner optically thick layers of the envelope, in which radiation is in equilibrium with matter, the radiative diffusion reduces both the radiation and internal gas energies. By day 116 the total optical depth of the ejecta falls to a value of $\sim 4$, and a low opacity of matter significantly weakens the interaction between radiation and matter. As a consequence, for the next 9 days the photon diffusion mainly reduces the stored radiation energy and eventually exhausts it, and the luminosity decreases abruptly and becomes close to the gamma-ray luminosity due to the decay of the radioactive ${ }^{56} \mathrm{Co}$, which is calculated by taking account of the retardation effect in the envelope transparent for gamma-rays (Fig. 13a).

\subsection{Plateau tail phase}

By the end of the previous phase, the bolometric luminosity does not fall directly to the gamma-ray luminosity (Fig. 13a), and the total radiation energy is not exhausted completely. Nearly at the same time the photosphere disappears, and the SN envelope becomes optically thin for radiation. For example, the total optical depth of the ejecta is 2.96 at the moment $t_{5}$, while the optical depth above the ${ }^{56} \mathrm{Ni}$ bubble shell is only 0.78 . Because during the phase of the radiation energy exhaustion the photon gas in the outer layers cools more intensively than in the inner part of the envelope ( $t=126$ and 130 days in Fig. 13b), the radiation flow is generated in the warm inner layers $(t=130$ and 134 days in Fig. 13b), then it propagates throughout the transparent layers, and results in some luminosity excess in the light curve just after the steep decline in the luminosity compared to the radioactive tail (Fig. 13a). We call this behavior of the light curve after the main plateau at the beginning of the radioactive tail "a plateau tail".

The radiation flow generated near the center of the envelope exists from $t \sim 130$ days to $t \sim 150$ days and then disappears (Fig. 13b). The characteristic duration of the plateau tail in the optimal model is 26 days (Fig. 13a). Note that the energy excess
Table 2. Hydrodynamic models based on the optimal model D11.

\begin{tabular}{cl}
\hline \hline Model & Remarks \\
\hline Dcc & no dense central core (Fig. 14a) \\
Ldn & less dense outer layers (Fig. 14c) \\
Mdn & more dense outer layers (Fig. 14c) \\
Hol & helium-rich composition in the outer layers: \\
& $X=0.368, Y=0.615$, and $Z=0.017$ (Fig. 15a) \\
Zol & low metallicity in the outer layers: \\
& $X=0.735, Y=0.259$, and $Z=0.006$ (Fig. 15c) \\
Sci & sharp metals/He/H composition interface (Fig. 16a) \\
Hec & helium core of about $8 M_{\odot}$ (Fig. $16 \mathrm{c}$ ) \\
Nhf & a half of ${ }^{56} \mathrm{Ni}$ amount: $M_{\mathrm{Ni}}=0.018 M_{\odot}$ \\
Nno & no radioactive ${ }^{56} \mathrm{Ni}$ \\
Nvm & ${ }^{56} \mathrm{Ni}$ mixed up to a velocity of $830 \mathrm{~km} \mathrm{~s}^{-1}$ \\
$\mathrm{Nvh}$ & ${ }^{56} \mathrm{Ni} \mathrm{mixed} \mathrm{up} \mathrm{to} \mathrm{a} \mathrm{velocity} \mathrm{of} 1095 \mathrm{~km} \mathrm{~s}^{-1}$ \\
Lop & no contribution of the expansion opacity \\
Ldk & without the limb-darkening effect \\
\hline
\end{tabular}

radiated during the plateau tail over the luminosity of radioactive decays is $2.5 \times 10^{46} \mathrm{erg}$ and $11 \%$ of the total radiation energy within the ejecta at the moment $t_{5}$.

\subsection{Radioactive tail of the light curve}

After the phase of the plateau tail, the bolometric luminosity of the optimal model decreases to the gamma-ray luminosity due to the decay of the radioactive ${ }^{56} \mathrm{Co}$ (Fig. 13a) and is solely powered by the radioactive energy source. This is how the stage of the radioactive tail starts, and the calculated light curve is in good agreement with the observations of SN 1999em after day 150 (Fig. 3). Note that the envelope remains optically thick for the gamma rays during a few hundred days (the total optical depth is 30.6 at $t=150$ days), and they deposit the bulk of their energy locally, at least at the beginning of the radioactive tail.

\section{General properties of the optimal model}

It is well known that the photometric characteristics of the SN IIP outburst are determined mainly by the basic parameters: the pre-SN radius $R_{0}$, the ejecta mass $M_{\text {env }}$, and the explosion energy $E$. Moreover, to get better agreement with the observations of SN $1999 \mathrm{em}$, we have to vary the density distribution in the pre-SN model, its chemical composition, the ${ }^{56} \mathrm{Ni}$ mass, and its mixing. A dependence of the bolometric light curve on the expansion opacity and the effect of limb darkening is of particular interest. These general properties of the optimal model are studied by means of the models listed in Table 2.

\subsection{Presupernova structure}

At first, we study the dependence of the bolometric luminosity on the inner layers' density in the pre-SN model and consider the extreme case of an auxiliary model similar to the optimal model but without a dense central core (Fig. 14a). In such a model the inner layers do less work moving outwards in the gravitational field than do those in the optimal model with a dense central core and hence acquire higher velocities than in the optimal model. In contrast, the outer layers of the optimal model expand faster than those of the auxiliary model. As a consequence, the photospheric radius of model D11 is greater than in the auxiliary model up to about day 35 and is smaller later on. At the comparable effective temperatures of the models, this results in completely different behavior by the bolometric light curves (Fig. 14b). This 

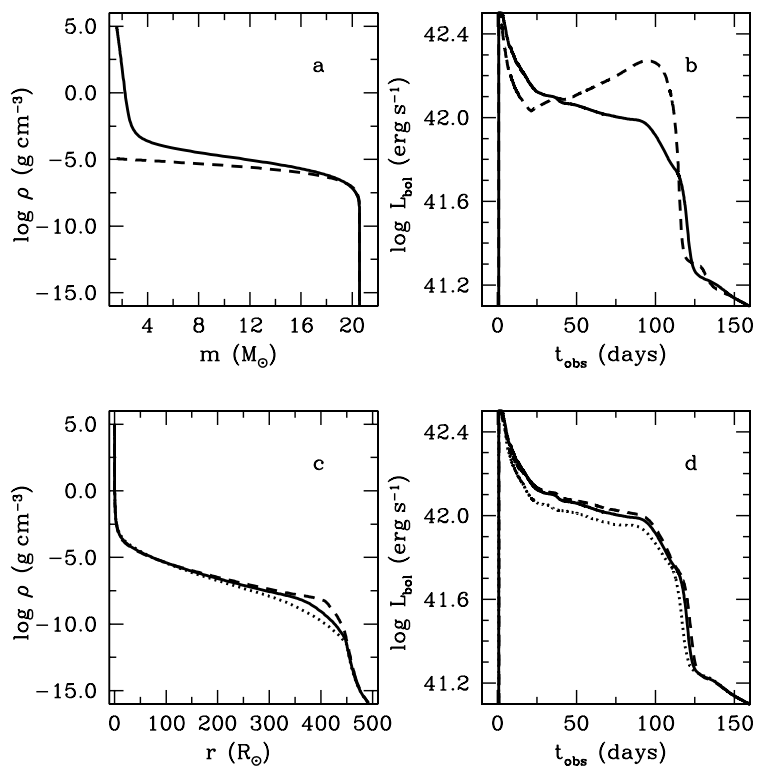

Fig. 14. Dependence on the pre-SN structure. Top: a) density distribution with respect to interior mass for the pre-SN model D11 (solid line) and the similar model Dcc but without a dense central core (dashed line), and b) the corresponding bolometric light curves. Bottom: c) density distribution with respect to radius for the pre-SN model D11 (solid line) and the similar models Ldn and Mdn, but with less (dotted line) and more (dashed line) dense outer layers, respectively, and d) the corresponding bolometric light curves.

implies that there is a dense central core inside the real pre-SN of SN 1999em.

In terms of the CRW properties the growth in the bolometric luminosity during the $\mathrm{CRW}$ phase in the auxiliary model (Fig. 14b) is controlled by effective index $n$ at the photosphere position that is greater by $\sim 3.5-0.5$ than in the optimal model. A greater effective index corresponds to a greater increase in the total flux of mass through the surface of the CRW front with time and, as a consequence, to faster growth in the bolometric luminosity with time (Grassberg \& Nadyozhin 1976).

The influence of the density distribution in the outer layers of the pre-SN on the bolometric luminosity is illustrated by model D11 and the same models but with both less dense and denser outer layers (Figs. 14c and 14d). A roughly similar behavior of the effective index $n$ at the photosphere position in these models reflects the approximately equal slopes of the corresponding bolometric light curves during the CRW phase. A transition from the model with the less dense outer layers to that of the denser layers is characterized by a reduction in the cooling by the adiabatic expansion and, consequently, by an increase in the bolometric luminosity in the whole SN outburst except for phases of the shock breakout and the radioactive tail. As a result, for these models the energies radiated during the first 180 days are $1.251 \times 10^{49} \mathrm{erg}, 1.355 \times 10^{49} \mathrm{erg}$, and $1.430 \times$ $10^{49} \mathrm{erg}$, respectively. Thus, the bolometric light curve is fairly sensitive to the initial structure of the outer layers.

\subsection{Chemical composition}

In addition to the pre-SN structure, the chemical composition of the ejecta also affects the SN luminosity. Let us consider a dependence on the chemical composition in the outer layers of the ejecta beyond the helium core. An enhancement of helium abundance at the expense of hydrogen abundance in model Hol
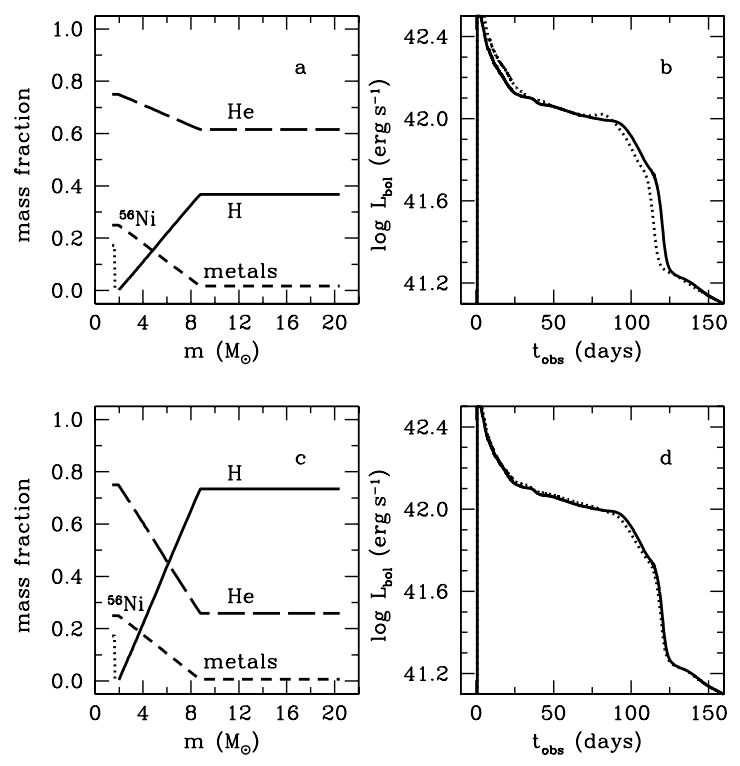

Fig. 15. Dependence on the chemical composition in the outer layers of the ejecta beyond the helium core. Top: a) the chemical composition of model Hol that differs from model D11 in the mass fraction of hydrogen (solid line) and helium (long dashed line) (Table 2), the distribution of heavy elements (short dashed line) and radioactive ${ }^{56} \mathrm{Ni}$ (dotted line) being the same, and b) the corresponding bolometric light curve (dotted line) and that of model D11 (solid line). Bottom: c) the chemical composition of model Zol that differs from model D11 by the mass fraction of helium and heavy elements (Table 2), and d) the corresponding bolometric light curve (dotted line) and that of model D11 (solid line).

(Fig. 15a and Table 2) results in the higher luminosity during the adiabatic cooling phase than that of model D11, nearly the same luminosity in the CRW phase, a small bump at the end of the plateau and then the shortened duration of the plateau (Fig. 15b). This behavior of the luminosity is due to the lower opacity in the outer layers of model Hol. On the other hand, lower metallicity for model Zol (Fig. 15c and Table 2) than for model D11 (Table 1) increases the bolometric luminosity slightly during the CRW phase and then reduces it somewhat at the phase of radiative diffusion cooling (Fig. 15d). This is explained by a smaller contribution of heavy elements to opacity in the outer layers of the ejecta in model Zol. Thus, the bolometric light curve depends weakly on the chemical composition in the outer layers of the ejecta beyond the helium core, while hydrogen is abundant there and controls opacity of matter.

A good fit of the calculated bolometric light curve for the optimal model to what is observed (Fig. 3) is obtained with mutual mixing of the hydrogen-rich and helium-rich matter in the inner layers of the ejecta (Fig. 2). It is appropriate to ask here in what way the sharp boundary between the hydrogen-rich and heliumrich layers at the edge of the helium core - a characteristic of the evolutionary models of pre-SNe - changes the light curve. In the auxiliary model Sci without the mutual mixing at the edge of the helium core (Table 2) this boundary is located at the interior mass of $5.4 M_{\odot}$ (Fig. 16a). It is known that a transition from the hydrogen-rich to helium-rich layers, i.e. from the low to high ionization potential matter, causes an increase in the effective temperature and, as a consequence, a growth in the bolometric luminosity (Grassberg \& Nadyozhin 1976; Utrobin 1989). In fact, when the CRW front reaches the sharp boundary between the hydrogen-rich and helium-rich layers, crosses it, and then enters the helium core, a distinct bump in the light curve at the end of the plateau appears (Fig. 16b). Evidently, this local 

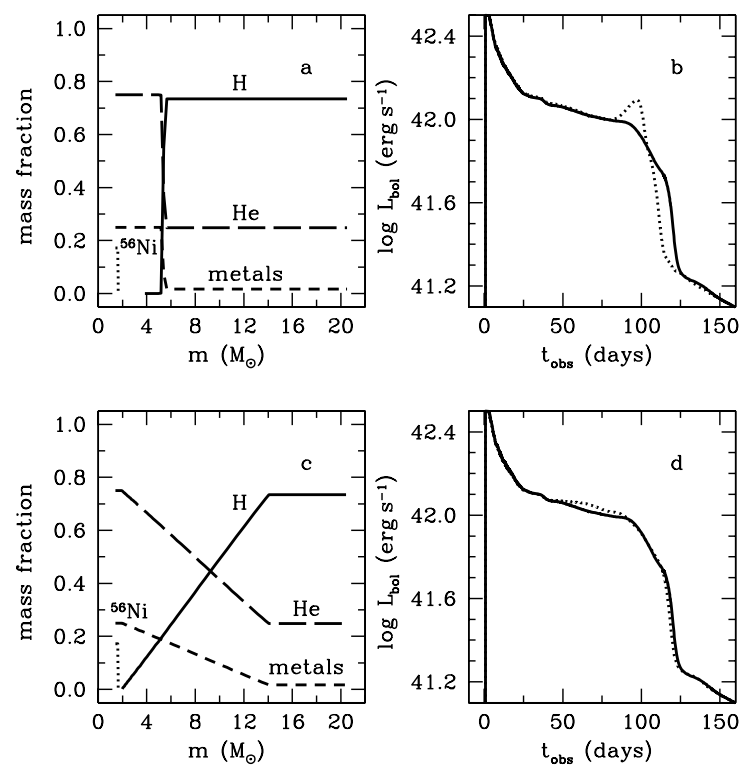

Fig. 16. Dependence on the chemical composition in the inner layers of the ejecta. Top: a) the chemical composition of model Sci, which differs from model D11 by the sharp metals/He/H composition interface, and b) the corresponding bolometric light curve (dotted line) and that of model D11 (solid line). Bottom: c) the chemical composition of model Hec, which differs from model D11 by the larger helium core, and d) the corresponding bolometric light curve (dotted line) and that of model D11 (solid line).

maximum of the luminosity is inconsistent with the observations of SN 1999em and other SNe IIP.

It is well known that at the time of an SN event the luminosity of a massive progenitor is determined mainly by the mass of the helium core, since a contribution of the hydrogen burning shell to the total energy generation is negligible. Hence the mass of the helium core is a critical quantity in evaluating the evolutionary state of a massive star. In this context it is intriguing that the increase in the helium core mass from 5.6 $M_{\odot}$ in model D1 1 to $8.1 M_{\odot}$ in model Hec (Fig. 16c), under a deep hydrogen mixing downward to $\approx 700 \mathrm{~km} \mathrm{~s}^{-1}$, leads to some decrease in the opacity of matter in the mixing region at the helium/hydrogen composition interface and, as a consequence, to the increase in the bolometric luminosity at the plateau (Fig. 16d). The resulting bolometric light curve is still consistent with the observations of SN 1999em. Thus, the mass of the helium core between $5.6 M_{\odot}$ and $8.1 M_{\odot}$ is acceptable for the optimal model.

\subsection{Mass of ${ }^{56} \mathrm{Ni}$ and its mixing}

After the CRW stage, when the radiative diffusion takes place, the radioactive decay of the ${ }^{56} \mathrm{Ni}$ and ${ }^{56} \mathrm{Co}$ nuclides begins to dominate in powering the luminosity. This fact is clearly demonstrated by model Nno without ${ }^{56} \mathrm{Ni}$ in the envelope (Fig. 17a and Table 2). A lack of ${ }^{56} \mathrm{Ni}$ shortens the duration of the bolometric light curve to about 100 days. It is evident that at later times the bolometric light curve depends on both the total mass of ${ }^{56} \mathrm{Ni}$ and its distribution over the ejecta. Of course, the total mass of ${ }^{56} \mathrm{Ni}$ is measured by the radioactive tail of the observed light curve and is $0.036 M_{\odot}$ in the optimal model for the Cepheid distance of 11.7 Mpc. A reduction in the total mass of ${ }^{56} \mathrm{Ni}$ from $0.036 M_{\odot}$ (model D11 in Table 1$)$ to $0.018 M_{\odot}$ (model Nhf in Table 2 ) results mainly in a shorter duration of the light curve (Fig. 17a).

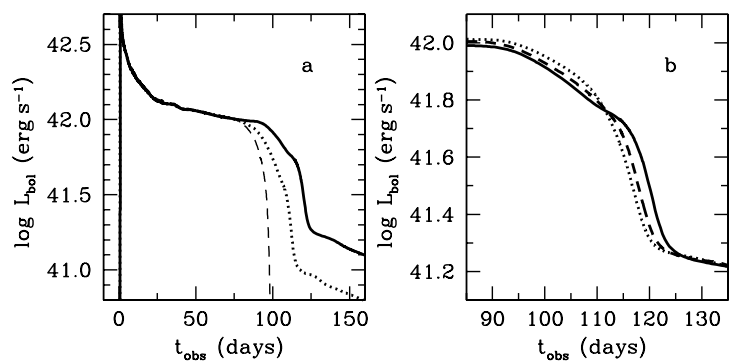

Fig. 17. Influence of the ${ }^{56} \mathrm{Ni}$ mass and its mixing. Panel a): bolometric light curves for model D11 (solid line) and the similar models Nhf and Nno but calculated with a half of the ${ }^{56} \mathrm{Ni}$ amount (dotted line) and without ${ }^{56} \mathrm{Ni}$ (dashed line), respectively. Panel b): bolometric light curves at the end of the plateau for model D11 (solid line), model Nvm (dashed line), and model Nvh (dotted line) in which ${ }^{56} \mathrm{Ni}$ is mixed up to a velocity of 660,830 , and $1095 \mathrm{~km} \mathrm{~s}^{-1}$, respectively.

Thus, the duration of the bolometric light curve has a minimum value of about 100 days and is a function of the ${ }^{56} \mathrm{Ni}$ amount.

To investigate the influence of the ${ }^{56} \mathrm{Ni}$ mixing on the light curve, hydrodynamic models Nvm and Nvh were calculated with the ${ }^{56} \mathrm{Ni}$ distribution different from that of model D11, but containing the same total mass of ${ }^{56} \mathrm{Ni}$ (Table 2). In these models the radioactive nickel is spread over a larger mass range of the ejected envelope or, equivalently, over a wider velocity range than in model D11. Hence, more favorable conditions for the radiative diffusion and an additional heating due to the radioactive decays should appear and therefore should increase the luminosity at the phase of radiative diffusion cooling that is clearly seen in Fig. 17b. In addition, this increase in the luminosity causes its subsequently earlier fall to the radioactive tail. As a result, with increasing the ${ }^{56} \mathrm{Ni}$ mixing in velocity space the linear luminosity shoulder at the phase of radiative diffusion cooling transforms into the convex light curve at the end of the plateau. Thus, a linear shoulder of the luminosity in logarithmic scale at the phase of radiative diffusion cooling implies a weak ${ }^{56} \mathrm{Ni}$ mixing. Because a discrepancy between the light curve of model D11 (which is in agreement with the observations of SN 1999em) and those of models Nvm and Nvh is rather significant, one can conclude that the bulk of the radioactive ${ }^{56} \mathrm{Ni}$ should be confined to the innermost layers of the ejected envelope expanding with velocities less than $660 \mathrm{~km} \mathrm{~s}^{-1}$.

\subsection{Plateau tail}

The general properties of the plateau tail on the radioactive tail are illustrated by the light curves shown in Fig. 18 and the relevant parameters of the models listed in Table 3 where $t_{5}$ is the time of the onset of the plateau tail as defined in Fig. 6 , $\Delta t^{2 \mathrm{p}}$ the characteristic duration of the plateau tail, $\Delta t_{\mathrm{R}}=R_{\text {ext }} / c$ is the characteristic time of radiation flow for the external radius of the expelled envelope $R_{\text {ext }}$ in the middle of the plateau tail, $\tau_{\text {tot }}$ the total optical depth of the ejecta at the moment $t_{5}$, $\Delta E_{\mathrm{r}}^{2 \mathrm{p}}$ the energy excess radiated during the plateau tail over the luminosity of radioactive decays, and $\Delta E_{\mathrm{r}}^{2 \mathrm{p}} / E_{\mathrm{r}}^{\mathrm{tot}}$ is a ratio of this energy to the total radiation energy within the ejecta at the moment $t_{5}$.

It is clear that there is no correlation between the characteristic duration of the plateau tail and the total optical depth of the ejecta (Table 3), and the duration depends very weakly on the opacity of matter (models D11 and Lop in Table 3). These regularities reflect the fact that the radiation responsible for the 

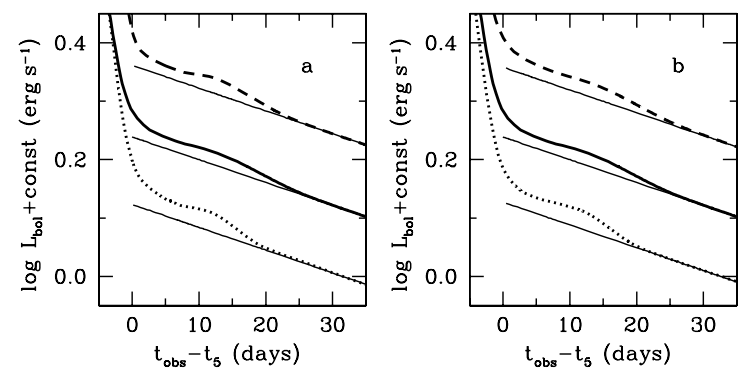

Fig. 18. Dependence of the plateau tail of the bolometric light curve on a) the ${ }^{56} \mathrm{Ni}$ amount (model $\mathrm{Nhf}$ in Table 2, dotted line) and the contribution of the expansion opacity (model Lop in Table 2, dashed line), and on b) the ejecta mass (model Mps in Table 4, dotted line) and the explosion energy (model Eps in Table 4, dashed line). Thick solid line is the bolometric light curve of model D11 and thin solid line is the luminosity of radioactive decays.

Table 3. Basic parameters at the phase of the plateau tail.

\begin{tabular}{ccccccccc}
\hline \hline Model & $\begin{array}{c}t_{5} \\
\text { (days) }\end{array}$ & $\begin{array}{c}\Delta t^{2 \mathrm{p}} \\
(\text { days })\end{array}$ & $\begin{array}{c}\Delta t_{\mathrm{R}} \\
(\text { days })\end{array}$ & $\Delta t^{2 \mathrm{p}} / \Delta t_{\mathrm{R}}$ & \multicolumn{4}{c}{$\begin{array}{c}\Delta E_{\mathrm{r}}^{2 \mathrm{p}} \\
\left(10^{46} \mathrm{erg}\right)\end{array}$} \\
\hline D11 & 124 & 26 & 8.76 & 2.97 & 2.96 & 2.5 & 0.110 \\
Lop & 110 & 25 & 7.83 & 3.19 & 1.71 & 3.8 & 0.136 \\
Nhf & 115 & 22 & 7.94 & 2.77 & 2.99 & 1.5 & 0.100 \\
Mps & 127 & 22 & 7.89 & 2.79 & 2.64 & 2.3 & 0.089 \\
Eps & 119 & 28 & 9.25 & 3.03 & 3.01 & 3.1 & 0.107 \\
\hline
\end{tabular}

plateau tail flows throughout an optically thin medium. On the other hand, the characteristic duration is proportional to the characteristic time of radiation flow independent of the parameters of the listed models (Table 3), and their ratio is 2.95 on average. It is worth noting that the energy excess of the plateau tail is roughly $10 \%$ of the total radiation energy at the moment $t_{5}$ for all but model Lop (Table 3).

The plateau tail of the bolometric light curve demonstrates the following important properties. A decrease in the ${ }^{56} \mathrm{Ni}$ mass (models D11 and Nhf) shortens the characteristic duration of the plateau tail and reduces its energy excess (Fig. 18a and Table 3). This influence of the ${ }^{56} \mathrm{Ni}$ amount is related to its large role in powering the light curve at the end of the main plateau after day 100 (Fig. 17a). The higher the average expansion velocity of the ejecta (models Mps, D11, and Eps), the longer the duration of the plateau tail and the higher the energy excess (Fig. 18b and Table 3). An increase in the duration of the plateau tail with the average expansion velocity results from its proportionality to the characteristic time of radiation flow. The higher average expansion velocity causes the onset of the plateau tail at an earlier time, when the total radiation energy is higher and, as a consequence, the energy excess, radiated over the luminosity of radioactive decays during the plateau tail, becomes greater.

\subsection{Expansion opacity}

A contribution of numerous metal lines to opacity plays a fundamental role in reproducing the observed light curve of SN 1999em, a normal SN IIP, as shown by the optimal model D11 and model Lop calculated by neglecting the expansion opacity (Fig. 19). The decrease in opacity due to disregarding the line contribution in model Lop speeds up the radiation diffusion and causes the bolometric luminosity to increase significantly compared to that of model D11 during nearly the whole outburst and, as a consequence, the characteristic

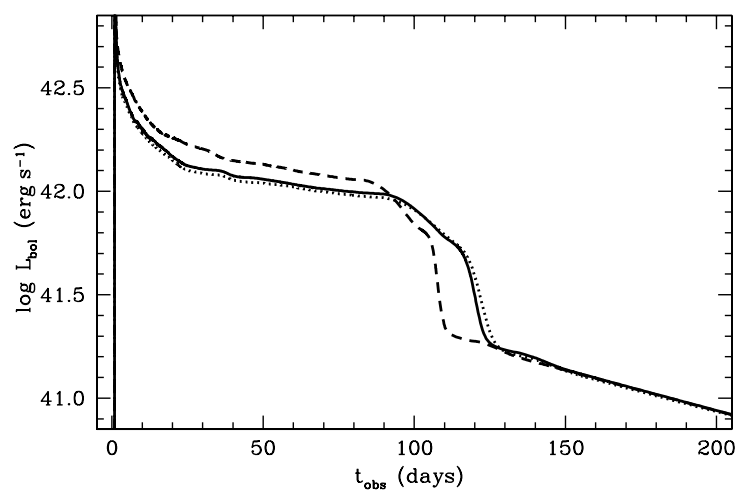

Fig. 19. The bolometric light curves for model D11 (solid line) and the similar models Lop and Ldk, but calculated without the expansion opacity (dashed line), and for the isotropic emergent radiation, without the limb darkening (dotted line).

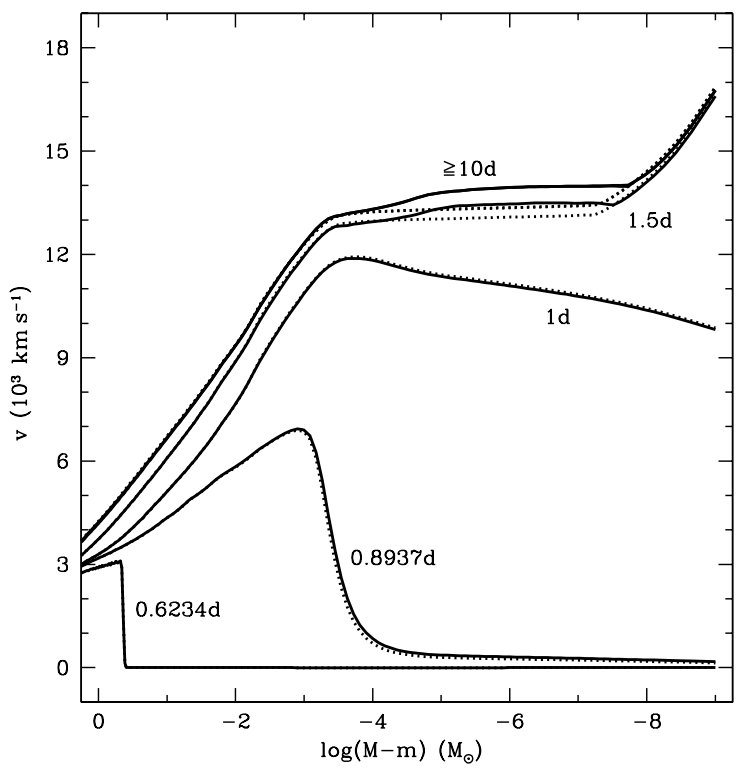

Fig. 20. Evolution of the velocity distribution in mass for models D11 (solid line) and Lop (dotted line): at $t=0.6234$ days when the shock approaches the pre-SN surface, at $t=0.8937$ days when the shock emerges on the surface, at $t=1$ days, at $t=1.5$ days, and subsequently, at later times $t \geq 10$ days when the envelope is already expanding homologously. The mass is measured from the envelope surface.

duration of the plateau to shorten by about 10 days. It is equivalent in action to an increase in the explosion energy that will be demonstrated in Sect. 7 (Fig. 22c). This fact leads to the firm conclusion that neglecting the expansion opacity underestimates the explosion energy and introduces an error of nearly $20 \%$ into its value.

It is worth noting that the slope of the light curve shoulder at the phase of radiative diffusion cooling in model D11 is flatter than in model Lop without the expansion opacity (Fig. 19). This behavior of the light curve is consistent with the dependence of the characteristic diffusion time on the optical depth of the diffusion region (1) which, in turn, is proportional to the opacity of matter.

The resonance scattering of radiation in numerous metal lines is also important in forming the gas flow in the outer layers of the expanding SN envelope (Fig. 20). After the shock emerges on the pre-SN surface at $t=0.8937$ days, an expansion of the envelope begins, and it becomes homologous during the next 10 days. An additional acceleration induced by the expansion 


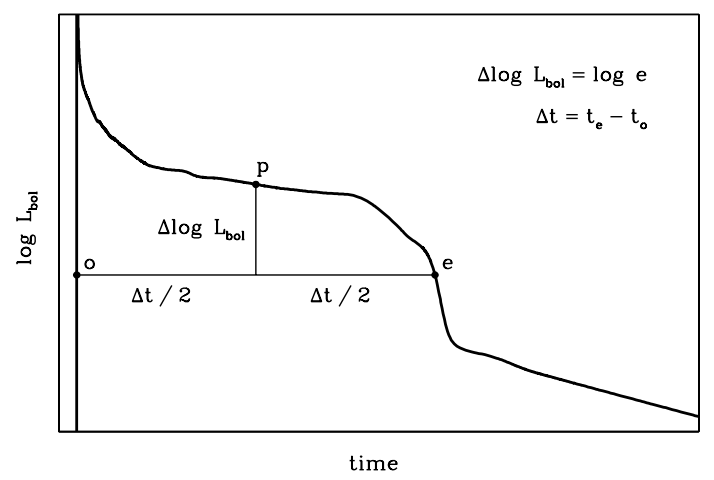

Fig. 21. Schematic bolometric light curve of SN IIP. Lettered dots mark the onset of the luminosity rising (o), the middle of the plateau (p), and the end of the plateau (e).

opacity occurs between $t \approx 1$ days and $t \approx 1.5$ days, lasts up to $t \approx 7.5$ days, and takes place in the outer layers with a mass of about $10^{-4} M_{\odot}$. A comparison of models D11 and Lop shows that these layers gain a velocity excess as large as $600 \mathrm{~km} \mathrm{~s}^{-1}$, except for the outermost layers with a mass of about $10^{-8} M_{\odot}$. Note that this additional acceleration is responsible for a feature in the gas flow as seen in Fig. 9 where the velocities of different mass shells in the ejected envelope as a function of time are overplotted on the photospheric velocity.

\subsection{Effect of limb darkening}

During the first 116 days, when a well-defined photosphere exists, particularly at the CRW phase, the emitted radiation is nearly isotropic, and the bolometric light curves of models D11 and Ldk, calculated without the effect of the limb darkening, almost coincide (Fig. 19). As the envelope expands and becomes optically thin, the continuum formation region gradually becomes more extended, and the degree of anisotropy of the emergent radiation increases. The increase in the radiation anisotropy with time clearly results in the difference between the light curves of models D11 and Ldk during the phase of the radiation energy exhaustion: the bolometric luminosity calculated by taking the limb-darkening law into account is lower than for isotropic radiation (Fig. 19).

Utrobin (2004) shows that the difference between the bolometric luminosity calculated by taking into account the emergent anisotropic radiation and the luminosity for the isotropic radiation depends on both the limb-darkening law and the retardation effect. Interestingly, this difference increases with growing the degree of the anisotropy of the emergent radiation, while its sign is determined solely by the time derivative of the bolometric luminosity. The latter property explains the behavior of the bolometric luminosity for models D11 and Ldk discussed above. It seems that in SN $1999 \mathrm{em}$, a normal SN IIP, the effect of limb darkening is more of purely academic interest than of practical value.

\section{Physical and observed parameters}

Generally, our main goal is to construct the adequate hydrodynamic model by fitting the photometric and spectroscopic observations of the object under study. Sometimes it is tempting to evaluate the relevant physical parameters for other $\mathrm{SN}$ with close observed properties. In this case, it is reasonable to use the results already obtained for the similar object. For this purpose, we

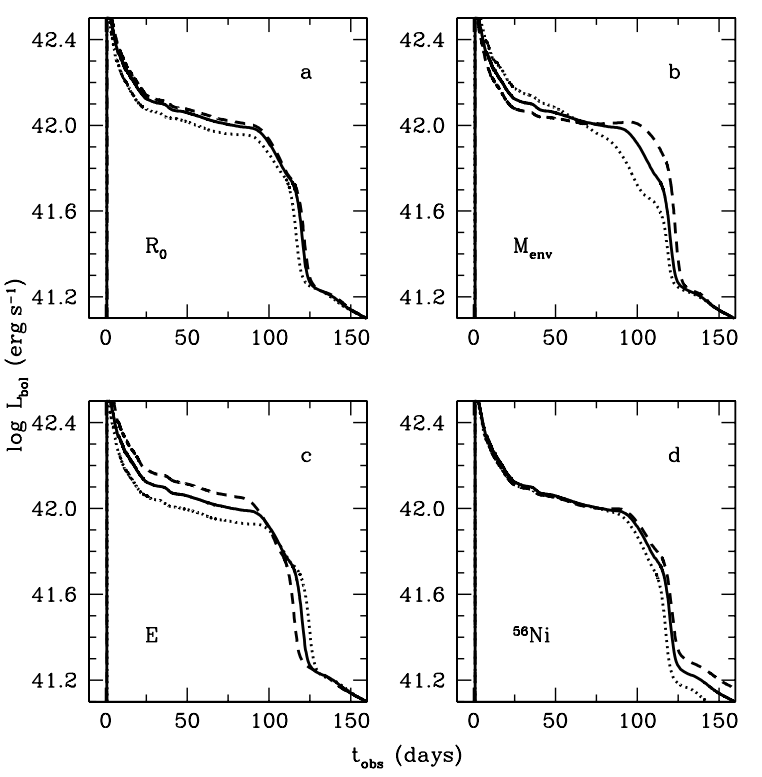

Fig. 22. Dependence of the bolometric light curve of the optimal model D11 (solid line) on the basic parameters: a) the initial radius, model Rms (dotted line) and model Rps (dashed line); b) the ejecta mass, model Mms (dotted line) and model Mps (dashed line); c) the explosion energy, model Ems (dotted line) and model Eps (dashed line); d) the total ${ }^{56} \mathrm{Ni}$ mass, model Nms (dotted line) and model Nps (dashed line).

have to find the relationships between the basic physical and observed parameters. Evidently, the important physical parameters are the initial radius $R_{0}$, the ejecta mass $M_{\text {env }}$, and the explosion energy $E$. The large impact of ${ }^{56} \mathrm{Ni}$ on the light curve near the end of the plateau, which is shown and discussed in Sect. 6.3, makes us take the total ${ }^{56} \mathrm{Ni}$ mass into account. Because of the temporal character of the energy deposition from radioactive decays, it is impossible to treat this energy input as an addition to the explosion energy, so we have to introduce the total ${ }^{56} \mathrm{Ni}$ mass $M_{\mathrm{Ni}}$ as the fourth basic physical parameter.

To describe the observed properties of SN IIP, Litvinova \& Nadyozhin $(1983,1985)$ used the three parameters: the plateau duration, the absolute $V$ magnitude, and the photospheric velocity in the middle of the plateau. However, in the course of time it became evident that this simple description needed to be corrected for the influence of ${ }^{56} \mathrm{Ni}$ on the light curve via an additional parameter (Nadyozhin 2003). Unfortunately, the bounds of the plateau are unclear because they are no specific points on the light curve. Moreover, the interval of a plateau duration misses the bolometric luminosity jump at the shock breakout and the subsequent adiabatic cooling phase together with the relevant dependence on the initial radius, the ejecta mass, and the explosion energy (Fig. 22). To overcome this problem, instead of the plateau duration we define a characteristic duration $\Delta t$ of the light curve as a whole, measuring it from the bolometric luminosity jump at the shock breakout to the end of the plateau for an $e$-fold reduction of the luminosity in the middle of plateau (Fig. 21). Of course, in this way the characteristic duration is easy to determine for a theoretical light curve, as shown in a schematic bolometric light curve of SN IIP, but very difficult for an observed one. Fortunately, this situation is not hopeless. First, it is possible to apply the theoretical light curve as a template for measuring the specific moments $t_{\mathrm{o}}$ and $t_{\mathrm{e}}$ (Fig. 21) and, consequently, for calculating the characteristic duration. Then, as the observational data become more and more extensive, the 
Table 4. Physical parameters of auxiliary hydrodynamic models.

\begin{tabular}{ccccccc}
\hline \hline Model & $\begin{array}{c}R_{0} \\
\left(R_{\odot}\right)\end{array}$ & $\begin{array}{c}M_{\text {env }} \\
\left(M_{\odot}\right)\end{array}$ & $\begin{array}{c}E \\
\left(10^{51} \mathrm{erg}\right)\end{array}$ & $\begin{array}{c}M_{\mathrm{Ni}} \\
\left(10^{-2} M_{\odot}\right)\end{array}$ & $X$ & $Z$ \\
\hline D11 & 500 & 19 & 1.30 & 3.60 & 0.735 & 0.017 \\
Rms & 425 & 19 & 1.30 & 3.60 & 0.735 & 0.017 \\
Rps & 575 & 19 & 1.30 & 3.60 & 0.735 & 0.017 \\
Mms & 500 & 16 & 1.30 & 3.60 & 0.735 & 0.017 \\
Mps & 500 & 22 & 1.30 & 3.60 & 0.735 & 0.017 \\
Ems & 500 & 19 & 1.10 & 3.60 & 0.735 & 0.017 \\
Eps & 500 & 19 & 1.50 & 3.60 & 0.735 & 0.017 \\
Nms & 500 & 19 & 1.30 & 3.06 & 0.735 & 0.017 \\
Nps & 500 & 19 & 1.30 & 4.14 & 0.735 & 0.017 \\
\hline
\end{tabular}

relevant templates may be constructed from the observed light curves of the well-studied SNe IIP. The second modification we made is to use the bolometric luminosity $L_{\mathrm{bol}}^{\mathrm{p}}$ in the middle of the plateau because it is more adequate than the absolute $V$ magnitude for estimating the explosion energy. The photospheric velocity $v_{\mathrm{ph}}^{\mathrm{p}}$ in the middle of the plateau is the third observed parameter in accordance with Litvinova \& Nadyozhin (1983, 1985).

To establish the relationships among the basic physical and observed parameters accepted above, we carried out a parameter study of hydrodynamic models varying the physical parameters and measuring the observed ones. Evidently, a four-parameter approximation is a crude description of the hydrodynamic model properties. The sensitivity of the bolometric light curve to the pre-SN structure and its chemical composition makes this approximation valid only for the vicinity of the optimal model D11, not for a whole region of the basic parameters. We restricted ourselves to a local parameter study in the vicinity of the optimal model and explored a $15 \%$ limited range of the basic physical parameters (Table 4). The resulting bolometric light curves of auxiliary hydrodynamic models are plotted in Fig. 22 and compared to the optimal model. The basic observed parameters of these models are evaluated according to the definition, given in Fig. 21, and listed in Table 5.

The influence of the basic physical parameters is illustrated by the bolometric light curves of the auxiliary models (Fig. 22). Increasing the initial radius causes an increase in the characteristic time of the envelope expansion and, as a consequence, an increase in the width of the narrow intense peak on the bolometric light curve produced by the heating of the outer layers at the shock breakout phase. In addition, an increase in the initial radius leads to a reduction in the cooling by the adiabatic expansion and, accordingly, to an increase in the bolometric luminosity during the whole SN outburst except for the phase of the radioactive tail (Fig. 22a). It is interesting that this behavior by the bolometric light curve with initial radius is similar to its dependence on the density structure in the outer layers (Fig. 14c), which is discussed in Sect. 6.1. The latter dependence may be considered as a dependence on some effective radius of the pre$\mathrm{SN}$. In other words, an increase in the density in the outer layers of the pre-SN mimics an increase in the initial radius. Both decreasing the ejecta mass and increasing the explosion energy enlarge the average expansion velocity of the envelope. The increase in the average velocity of the envelope makes the shock wave propagating through the pre-SN matter stronger and, as a result, it heats the matter to a higher temperature, which increases the luminosity in the narrow peak of the light curve, at the subsequent phase of adiabatic cooling and at the beginning of the CRW phase (Figs. 22b and 22c). By the end of the
Table 5. Observed parameters of auxiliary hydrodynamic models.

\begin{tabular}{cccccc}
\hline \hline Model & $\begin{array}{c}\Delta t \\
(\text { days })\end{array}$ & $\begin{array}{c}\log L_{\text {bol }}^{\mathrm{p}} \\
\left(\mathrm{erg} \mathrm{s}^{-1}\right)\end{array}$ & $\begin{array}{c}v_{\mathrm{ph}}^{\mathrm{p}} \\
\left(\mathrm{km} \mathrm{s}^{-1}\right)\end{array}$ & $q_{\mathrm{kin}}$ & $q_{\mathrm{rad}}$ \\
\hline D11 & 117.57 & 42.0350 & 2374.4 & 0.8541 & 0.8258 \\
Rms & 114.88 & 41.9927 & 2335.3 & 0.8088 & 0.8429 \\
Rps & 118.25 & 42.0521 & 2388.3 & 0.8354 & 0.7826 \\
Mms & 111.70 & 42.0542 & 2481.5 & 0.7767 & 0.7881 \\
Mps & 121.64 & 42.0170 & 2291.9 & 0.9141 & 0.8252 \\
Ems & 122.50 & 41.9629 & 2134.7 & 0.8134 & 0.8040 \\
Eps & 111.93 & 42.0979 & 2640.5 & 0.8890 & 0.8424 \\
Nms & 113.98 & 42.0372 & 2452.5 & 0.8943 & 0.8303 \\
Nps & 119.39 & 42.0264 & 2320.3 & 0.7968 & 0.8102 \\
\hline
\end{tabular}

CRW phase, decreasing the ejecta mass reduces the bolometric luminosity and shortens the characteristic duration of the light curve (Fig. 22b). In contrast, increasing the explosion energy results in the luminosity growing and, accordingly, in shortening the characteristic duration as well (Fig. 22c). Up to the phase of the radiative diffusion cooling, the radioactive ${ }^{56} \mathrm{Ni}$ does not affect the light curve; but during this phase and later on, the energy deposition from radioactive decays powers the luminosity (Fig. 22d). The higher the total ${ }^{56} \mathrm{Ni}$ mass, the greater the energy deposition and the later the luminosity descending to the radioactive tail. Note that a slope of the luminosity shoulder at the phase of the radiative diffusion cooling clearly depends on the initial radius (Fig. 22a) and on the explosion energy (Fig. 22c) in a qualitative agreement with the simple description of photon diffusion (1).

To evaluate the three basic physical parameters (the initial radius, the ejecta mass, and the explosion energy) from the four values measured from observations (the characteristic duration of the light curve, the bolometric luminosity and the photospheric velocity in the middle of the plateau, and the total ${ }^{56} \mathrm{Ni}$ mass), we use the following approximate relations:

$$
\begin{aligned}
\log R_{0}= & +3.481 \log L_{\mathrm{bol}}^{\mathrm{p}}-5.937 \log v_{\mathrm{ph}}^{\mathrm{p}}-1.999 \log \Delta t \\
& -0.499 \log M_{\mathrm{Ni}}-120.174
\end{aligned}
$$

$\log M_{\mathrm{env}}=-2.942 \log L_{\mathrm{bol}}^{\mathrm{p}}+7.606 \log v_{\mathrm{ph}}^{\mathrm{p}}+7.807 \log \Delta t$ $-0.042 \log M_{\mathrm{Ni}}+83.045$,

$\log E=-1.476 \log L_{\mathrm{bol}}^{\mathrm{p}}+4.899 \log v_{\mathrm{ph}}^{\mathrm{p}}+3.040 \log \Delta t$

$$
+0.312 \log M_{\mathrm{Ni}}+90.765,
$$

where $R_{0}, M_{\mathrm{env}}$, and $M_{\mathrm{Ni}}$ are in solar units, $E$ in units of erg, $L_{\mathrm{bol}}^{\mathrm{p}}$ in $\mathrm{erg} \mathrm{s}^{-1}, v_{\mathrm{ph}}^{\mathrm{p}}$ in $\mathrm{km} \mathrm{s}^{-1}$, and $\Delta t$ in days. The above approximate formulae (2-4) are found by means of minimizing the errors between the values calculated with these relations and the corresponding physical parameters from Table 4 . For this purpose we constructed a variational functional in a quadratic form depending on the functional values, i.e. coefficients in the approximate formulae (2-4), and then used the direct search method of Powell (1964). Note that the obtained approximate formulae give the estimates of the physical parameters for the auxiliary models, the accurate values of which are listed in Table 4, with an error as great as $\approx 2.5 \%$.

In addition to the observed parameters of the auxiliary models, the important energetic ratios $q_{\text {kin }}=0.5 M_{\mathrm{env}} v_{\mathrm{ph}}^{\mathrm{p}}{ }^{2} / E_{\mathrm{kin}}$ and $q_{\mathrm{rad}}=L_{\mathrm{bol}}^{\mathrm{p}} \Delta t / E_{\mathrm{rad}}$, where $E_{\mathrm{kin}}$ is the kinetic energy of the ejecta and $E_{\text {rad }}$ is the total radiation energy emitted during the first 
Table 6. Hydrodynamic models for SN 1999em and SN 1987A.

\begin{tabular}{cccccccc}
\hline \hline SN & $\begin{array}{c}R_{0} \\
\left(R_{\odot}\right)\end{array}$ & $\begin{array}{c}M_{\text {env }} \\
\left(M_{\odot}\right)\end{array}$ & $\begin{array}{c}E \\
\left(10^{51} \mathrm{erg}\right)\end{array}$ & $\begin{array}{c}M_{\mathrm{Ni}} \\
\left(10^{-2} M_{\odot}\right)\end{array}$ & $Z$ & $\begin{array}{c}v_{\mathrm{Ni}}^{\max } \\
\left(\mathrm{km} \mathrm{s}^{-1}\right)\end{array}$ & $\begin{array}{c}v_{\mathrm{H}}^{\min } \\
\left(\mathrm{km} \mathrm{s}^{-1}\right)\end{array}$ \\
\hline 99em & 500 & 19 & 1.3 & 3.60 & 0.017 & 660 & 700 \\
$87 \mathrm{~A}$ & 35 & 18 & 1.5 & 7.65 & 0.006 & 3000 & 600 \\
\hline
\end{tabular}

180 days, are given in Table 5. The mean values of the ratios $q_{\mathrm{kin}}$ and $q_{\mathrm{rad}}$ are 0.842 and 0.816 with the standard deviations of $4.4 \%$ and $2.3 \%$, respectively. A quite low value of the standard deviation in the energetic ratios shows that the kinetic energy of the envelope and the total radiation energy are approximated more or less accurately by the observed parameters: $L_{\mathrm{bol}}^{\mathrm{p}}, v_{\mathrm{ph}}^{\mathrm{p}}$, and $\Delta t$.

A functional dependence of the observed parameters on the physical ones is, in turn, derived directly from the approximate formulae (2-4) and can be written as

$$
\begin{aligned}
\log L_{\mathrm{bol}}^{\mathrm{p}}= & +0.488 \log R_{0}-0.267 \log M_{\mathrm{env}}+1.006 \log E \\
& -0.082 \log M_{\mathrm{Ni}}-10.469,
\end{aligned}
$$

$$
\begin{aligned}
\log v_{\mathrm{ph}}^{\mathrm{p}}= & +0.083 \log R_{0}-0.247 \log M_{\mathrm{env}}+0.688 \log E \\
& -0.184 \log M_{\mathrm{Ni}}-31.951,
\end{aligned}
$$

$$
\begin{aligned}
\log \Delta t= & +0.103 \log R_{0}+0.268 \log M_{\mathrm{env}}-0.291 \log E \\
& +0.154 \log M_{\mathrm{Ni}}+16.548
\end{aligned}
$$

The estimates of the observed parameters for the auxiliary models obtained with the above relations (5-7) differ from the accurate values given in Table 5 by an error less than $\approx 2.2 \%$.

Finally, it should be emphasized that all of the above formulae approximate the physical parameters and the observable properties of hydrodynamic models but only in the vicinity of the optimal model D11. To cover a wider range of the parameters, we have to construct a global approximation, which should be based, in our opinion, on a set of hydrodynamic models of many real SNe IIP, but not on that of arbitrary hydrodynamic models.

\section{Comparison with SN 1987A}

Now we have got a confidence in reproducing the observed bolometric light curve and the $\mathrm{H} \alpha$ line of SN 1999em, a normal SN IIP, we can compare this object with the well-studied SN 1987A, a peculiar SN IIP. The important parameters of the optimal model D11 for SN 1999em and model M18 for SN 1987A (Utrobin 2005) are given in Table 6. It is remarkable that the ejecta mass $M_{\text {env }}$, the explosion energy $E$, and the minimum velocity of the hydrogen-rich envelope $v_{\mathrm{H}}^{\min }$ are comparable, while the pre-SN radius $R_{0}$, the mass fraction of heavy elements in the outer layers of the pre-SN $Z$, the total mass of ${ }^{56} \mathrm{Ni} M_{\mathrm{Ni}}$, and its maximum velocity $v_{\mathrm{Ni}}^{\max }$ are quite different.

The mass of a star is a fundamental parameter that determines the properties of the star and the course of its evolution. The pre-SN masses of SN 1999em and SN 1987A, estimated by the mass of the neutron star and the ejecta mass, are nearly $20.6 M_{\odot}$ and $19.6 M_{\odot}$, respectively. The masses of helium cores in the pre-SNe of SN 1999em and SN 1987A are 5.6-8.1 $M_{\odot}$ and $6.0 M_{\odot}$ (Woosley 1988), respectively. These masses are close enough to suppose that, in the final stages of stellar evolution, nearly the same iron cores form within the pre-SNe. This fact and roughly the same explosion energies of SN 1999em and
Table 7. Shock breakout and UV flash in SN 1999em and SN 1987A.

\begin{tabular}{ccccccccc}
\hline \hline SN & $\begin{array}{c}t_{\mathrm{sh}} \\
(\text { days })\end{array}$ & $\begin{array}{c}T_{\mathrm{c}}^{\max } \\
\left(10^{5} \mathrm{~K}\right)\end{array}$ & $\begin{array}{c}T_{\mathrm{eff}}^{\max } \\
\left(10^{5} \mathrm{~K}\right)\end{array}$ & $\begin{array}{c}\log L_{\mathrm{bol}}^{\max } \\
\left(\mathrm{erg} \mathrm{s}^{-1}\right)\end{array}$ & $\begin{array}{c}\Delta t_{L} \\
(\text { days })\end{array}$ & $\begin{array}{c}N^{\text {tot }} \\
\left(10^{56}\right)\end{array}$ & $\begin{array}{c}t^{N} \\
(\text { days })\end{array}$ & $\begin{array}{c}E_{\mathrm{rad}}^{N} \\
\left(10^{46} \mathrm{erg}\right)\end{array}$ \\
\hline 99em & 0.865 & 3.84 & 1.76 & 44.81 & 0.0200 & 276.8 & 1.234 & 159 \\
$87 \mathrm{~A}$ & 0.049 & 13.2 & 5.68 & 44.63 & 0.0007 & 7.615 & 0.266 & 6.24 \\
\hline
\end{tabular}

SN 1987A imply a unique explosion mechanism for these core collapse SNe.

It is evident that the amount of radioactive ${ }^{56} \mathrm{Ni}$ and its distribution in the SN envelope are a clear trace left by the explosion mechanism. Both SN 1999em and SN 1987A differ in the total mass of radioactive ${ }^{56} \mathrm{Ni}$ and in its distribution throughout the envelope (Table 6). Note that for these SNe the total ${ }^{56} \mathrm{Ni}$ mass is correlated to the explosion energy. This dependence is consistent with the empirical correlation between the total ${ }^{56} \mathrm{Ni}$ mass and the explosion energy for SNe IIP found by Nadyozhin (2003) and Hamuy (2003). The optimal model for SN $1999 \mathrm{em}$ is characterized by a weak ${ }^{56} \mathrm{Ni}$ mixing in velocity space up to $\approx 660 \mathrm{~km} \mathrm{~s}^{-1}$ and a deep hydrogen mixing downward to $\approx 700 \mathrm{~km} \mathrm{~s}^{-1}$ (Fig. 2 and Table 6 ). In turn, the hydrodynamic model for SN 1987A is characterized by a moderate ${ }^{56} \mathrm{Ni}$ mixing up to $\sim 3000 \mathrm{~km} \mathrm{~s}^{-1}$ and a deep hydrogen mixing downward to $\sim 600 \mathrm{~km} \mathrm{~s}^{-1}$ (Utrobin 2005).

The structure of the pre-SN and the chemical composition of its outer layers, which are unaffected by explosive nucleosynthesis and which are the end result of the entire evolution of the star, determine the pattern of SN outburst in many respects. The pre-SN model for SN 1999em has the structure of a red supergiant with a radius of $500 R_{\odot}$, and the chemical composition of its outer layers is the standard solar composition. It is quite definite that the progenitor of SN 1987A was the star Sanduleak $-69^{\circ} 202$, a B3 Ia blue supergiant. The pre-SN model for SN 1987A has a radius of $35 R_{\odot}$, and the chemical composition of its outer layers is typical of the LMC chemical composition, $X=0.743, Y=0.251$, and $Z=0.006$ (Dufour 1984). It is worth noting that a deficit of heavy elements in the LMC matter compared to the standard solar composition favors the formation of blue supergiants (Arnett 1987; Hillebrandt et al. 1987). In the case of SN 1987A, a relative compactness of the pre-SN is a major factor in understanding the peculiar properties of this phenomenon (Grassberg et al. 1987).

The basic characteristics of the shock breakout and the UV flash in SN 1999em and SN 1987A are summarized in Table 7 where $t_{\mathrm{sh}}$ is the time when the shock wave reaches the stellar surface, $T_{\mathrm{c}}^{\max }$ the maximum of the color temperature, $T_{\mathrm{eff}}^{\max }$ the maximum of the effective temperature, $L_{\mathrm{bol}}^{\max }$ the maximum of the bolometric luminosity, $\Delta t_{L}$ the width of the luminosity peak at a half level of its maximum, $N^{\text {tot }}$ the total number of ionizing photons above $13.598 \mathrm{eV}$ for the whole outburst, $t^{N}$ the time when the number of ionizing photons measures up $90 \%$ of the total number, and $E_{\text {rad }}^{N}$ the energy radiated by the $S N$ during this time.

Under the comparable ejecta masses and the explosion energies, the smaller the pre-SN radius, the higher the velocity of matter in the outermost layers with a sharp decline of density where the matter accelerates due to the effect of hydrodynamic cumulation. At the same time the characteristic time of the envelope expansion becomes shorter and the adiabatic losses of energy greater. Clearly, in SN 1999em the shock wave reaches the stellar surface much later than in SN 1987A, and the color and effective temperatures jump to lower values, while the bolometric luminosity rises to a higher value and its peak is much wider 


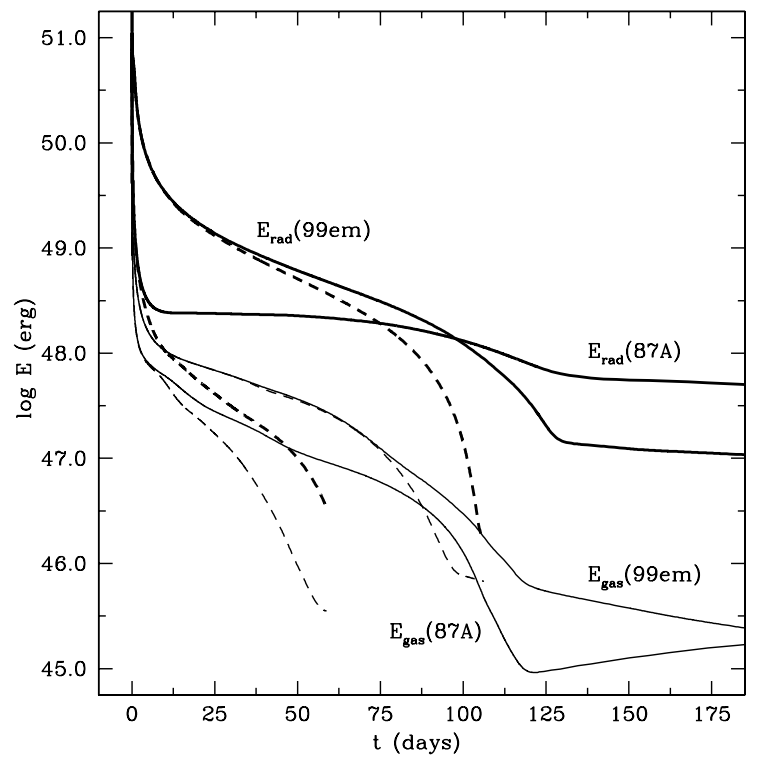

Fig. 23. Total radiation (thick solid line) and gas (thin solid line) energies as a function of time for SN 1999em and SN 1987A. The dependence on the energy deposition from radioactive decays is shown by the special hydrodynamic models without ${ }^{56} \mathrm{Ni}$ in the envelope (dashed line).

(Table 7). Note that in SN 1999em the bolometric luminosity peak coincides with the maximum of the effective temperature and occurs after the shock wave reaches the stellar surface in contrast to SN 1987A in which the maximum of the effective temperature precedes the peak, and the shock wave emerges between them on the pre-SN surface. The UV flash in SN 1999em produces a greater total number of ionizing photons by a factor of 36.3, and it lasts longer than in SN 1987A by a factor of 4.6, the radiated energy being greater by a factor of 25.5 (Table 7).

The smaller the pre-SN radius, the greater the adiabatic losses of energy and the shorter the CRW phase - a characteristic feature of SNe IIP. A difference between the explosions of the red and blue supergiants is radical in the total radiation and gas energies as a function of time (Fig. 23) and, especially, in the light curves (Fig. 24). To clarify the physical processes in SN 1999em and SN 1987A, we compare the optimal models of these $\mathrm{SNe}$ with the special hydrodynamic models containing no ${ }^{56} \mathrm{Ni}$ in the envelope. Note that during the entire outburst the total radiation energy is much greater than the total gas energy of the envelope. A lack of ${ }^{56} \mathrm{Ni}$ makes the energy losses due to the adiabatic expansion a dominant process controlling the energy balance of an SN. In this case, the blue supergiant structure of the pre-SN for SN 1987A results in such huge energy losses that its total radiation energy is much less than that of SN 1999em the whole time and is completely exhausted in about 50 days, while for SN 1999em this happens later by around 50 days. Accordingly, the luminosity at the CRW phase is maintained by the stored energy during only 22 days for SN 1987A and about 75 days for SN $1999 \mathrm{em}$.

The subsequent run of the bolometric light curves in the optimal models of these SNe is entirely determined by the energy deposition of gamma rays from the radioactive decays and shows the key role played by ${ }^{56} \mathrm{Ni}$ and ${ }^{56} \mathrm{Co}$ in powering the light curve (Fig. 24). The luminosity of SN 1999em exceeds the gammaray luminosity from the peak to the radioactive tail. On the contrary, the luminosity of SN 1987A drops abruptly well below the gamma-ray luminosity during the adiabatic cooling phase, grows

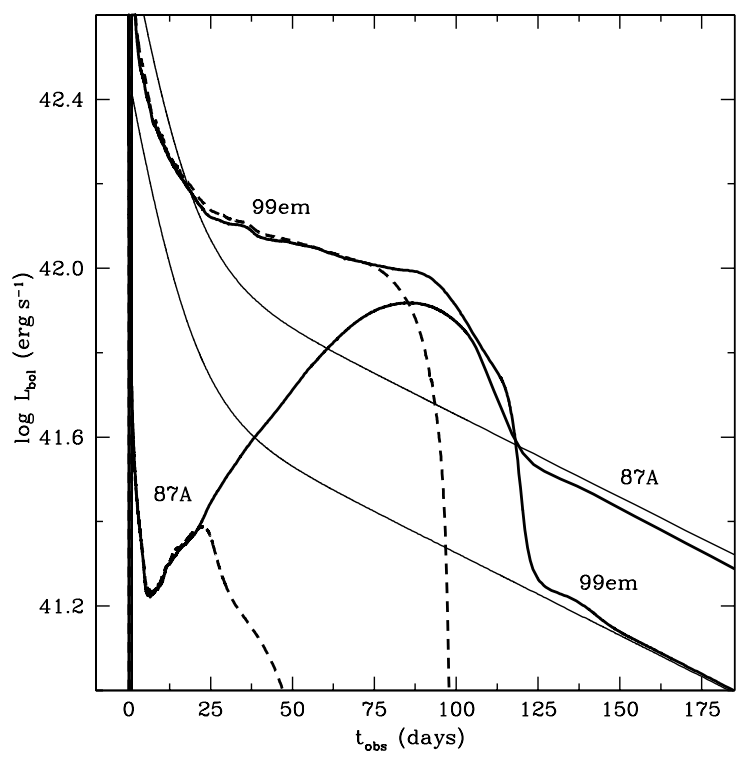

Fig. 24. Comparison of the calculated bolometric light curve (thick solid line) with the gamma-ray luminosity (thin solid line) for SN 1999em and SN 1987A. The special hydrodynamic models containing no ${ }^{56} \mathrm{Ni}$ in the envelope show the influence of the energy deposition from radioactive decays (dashed line).

to the maximum after the CRW phase in about 65 days exceeding the latter at day 60 , and forms a wide dome on the light curve, declining to the radioactive tail and subsequently diving under it. The wide dome on the light curve of the SN 1987A is physically equivalent to the phases of the radiative diffusion cooling, the exhaustion of radiation energy, and the plateau tail in the evolution of SN 1999em in the sense that it contains the basic features of all these phases.

At the stage of the radioactive tail, there is a striking difference between SN 1999em and SN 1987A: the internal gas energy of the envelope decreases with time in SN 1999em and increases in SN 1987A (Fig. 23); the bolometric luminosity of SN 1999 em coincides with the corresponding gamma-ray luminosity, while the bolometric luminosity of SN 1987A runs below it (Fig. 24). The gas energy is controlled by the energy rate of gamma-ray deposition, the energy losses due to the adiabatic expansion, and the net rate of absorption-emission processes. At a given phase the last is dominated by the emission processes. In the envelope of SN 1999em, the adiabatic energy losses are much greater than those in the envelope of SN 1987A where the thermal gas energy is much less than that of SN 1999em. This fact explains both the decrease in the total gas energy with time in SN 1999em and its increase in SN 1987A.

The radiation energy is ruled mainly by the energy rate of gamma-ray deposition and the rate of work done by radiation pressure. In the envelope of SN 1999em the total radiation energy is much less than that in the envelope of SN 1987A, and, consequently, the rate of work done by radiation pressure in SN 1999em is much less than that in SN 1987A as well. It leads to a negligible difference between the bolometric and gammaray light curves in the optimal model of SN 1999em and to the bolometric light curve running below the gamma-ray light curve in the optimal model of SN 1987A. Note that the higher a rate of work done by radiation pressure in an SN envelope, the larger the excess of the gamma-ray luminosity over the bolometric one at the radioactive tail when the $\mathrm{SN}$ envelope still remains optically thick for gamma rays. In the optimal model of SN 1987A, 
the excess is nearly $7 \%$ (Fig. 24), which corresponds to an underestimation of the ${ }^{56} \mathrm{Ni}$ amount of the same value in equating the observed bolometric luminosity to the gamma-ray one. Such an excess of the gamma-ray luminosity over the bolometric one is of great interest and should be taken into account in measuring the ${ }^{56} \mathrm{Ni}$ amount from the observed bolometric luminosity at the radioactive tail.

It is very important that the approximation of homologous expansion may be used in the atmosphere models starting from nearly day 2.8 (Sect. 5.3) for SN 1999em, a normal SN IIP, and from day 1 (Utrobin 2004) for SN 1987A, a peculiar SN IIP. Because of higher expansion velocities in the ejecta, the effects of expansion opacity and limb darkening are more prominent in SN 1987A than in SN 1999em. For both SNe IIP, the crucial role of the time-dependent approach in atmosphere models at the photospheric epoch is evident from a comparison of spectral lines computed in this approach with those in the steady-state approximation.

\section{Discussion}

The aim of this paper is to explore the SN 1999em event, a normal SN IIP, by comparing the hydrodynamic models and the time-dependent atmosphere models with both the photometric and spectroscopic observations. The resulting optimal model of SN 1999em succeeds in reproducing the observed features. The bolometric light curve and the spectral evolution of the $\mathrm{H} \alpha$ line are consistent with a radius of the pre-SN of $500 R_{\odot}$, a mass of the ejected envelope of $19 M_{\odot}$, an explosion energy of $1.3 \times$ $10^{51} \mathrm{erg}$, and a mass of radioactive ${ }^{56} \mathrm{Ni}$ of $0.036 M_{\odot}$, the bulk of which is confined to layers ejected with velocities less than $\approx 660 \mathrm{~km} \mathrm{~s}^{-1}$.

The observational data of an SN are measured with some errors that yield the uncertainties in values inferred from them. In the case of SN $1999 \mathrm{em}$, we can translate these measurement errors of the observed parameters into uncertainties of the physical parameters using the approximate formulae (2-4). To assign an uncertainty to the bolometric luminosity in the middle of the plateau, we adopt a relative error of $7 \%$ for measuring the bolometric luminosity itself (Elmhamdi et al. 2003) and an uncertainty of $\pm 1 \mathrm{Mpc}$ for the Cepheid distance to the host galaxy (Leonard et al. 2003). The resulting relative error in the bolometric luminosity in the middle of the plateau is $26 \%$, as well as the error in the mass of radioactive ${ }^{56} \mathrm{Ni}$. The relative errors in the photospheric velocities do not exceed 5\%, inferred from the Fe II 4924, 5018, and $5169 \AA$ absorption lines in the middle of the plateau (Hamuy et al. 2001; Leonard et al. 2002). The scatter in the explosion date yielded by the EPM varies within \pm 2 days (Hamuy et al. 2001; Leonard et al. 2002; Elmhamdi et al. 2003). To estimate the measurement errors in the characteristic duration of the light curve, we consequently adopted an uncertainty of \pm 4 days. All the accepted errors in the observed values yield the following uncertainties in physical parameters: $\pm 200 R_{\odot}$ in the pre-SN radius, $\pm 1.2 M_{\odot}$ in the ejecta mass, $\pm 0.1 \times 10^{51} \mathrm{erg}$ in the explosion energy, and $\pm 0.009 M_{\odot}$ in the mass of radioactive ${ }^{56} \mathrm{Ni}$.

Elmhamdi et al. (2003) note a clear flattening in the $B V R I$ light curves of SN 1999em after the main plateau at the beginning of the radioactive tail and call it "the second plateau". The "UBVRI" bolometric light curve of SN 1999em consequently reflects the second plateau. The observed second plateau coincides in time with the plateau tail of the bolometric light curve for the optimal model (Sect. 5.7), but runs slightly below a line of the radioactive tail in contrast to the latter. It seems that the real bolometric luminosity of SN $1999 \mathrm{em}$ is greater than the " $U B V R I$ ' luminosity during the second plateau. Elmhamdi et al. (2003) also report on the second plateau on the tail for SN 1991G and SN 1997D, and find that its duration is correlated with the amount of ejected ${ }^{56} \mathrm{Ni}$, as well as the duration of the plateau tail does. Of course, a second plateau nature that is different from that of the plateau tail cannot be excluded at the present time.

In the same study, Elmhamdi et al. explore the width and position of the He I $10830 \AA$ line, which is sensitive to the nonthermal ionization and excitation produced by the radioactive decays, and show that in the ejecta of SN 1999em the bulk of ${ }^{56} \mathrm{Ni}$ was distributed inside a region with a velocity less than $1100 \mathrm{~km} \mathrm{~s}^{-1}$ in the close hemisphere. Obtained in the optimal model, a weak ${ }^{56} \mathrm{Ni}$ mixing in velocity space up to $\approx 660 \mathrm{~km} \mathrm{~s}^{-1}$ agrees well with this ${ }^{56} \mathrm{Ni}$ distribution. By interpreting the temporal evolution of the [O I] 6300, $6364 \AA$ doublet profile in terms of the dust formation, they conclude that the dust occupies a sphere with a velocity of $\approx 800 \mathrm{~km} \mathrm{~s}^{-1}$. Dust grains are formed by heavy elements, so we should estimate the volume containing them. The characteristic velocity of the outer edge of the metal-rich region is nearly $850 \mathrm{~km} \mathrm{~s}^{-1}$ in the optimal model. Moreover, the ${ }^{56} \mathrm{Ni}$ bubble shell containing heavy elements is unstable to the Rayleigh-Taylor instability and, consequently, should be broken by it (Basko 1994) and mixed, thereby producing dense clumps in which dust can form. Thus, inside the sphere of a velocity of roughly $850 \mathrm{~km} \mathrm{~s}^{-1}$ in the optimal model of SN $1999 \mathrm{em}$, there are conditions that favor the dust formation in the ejecta at late times. In addition, from the observed [O I] 6300, $6364 \AA$ doublet luminosity, they infer the oxygen mass of $\sim 0.3-0.4 M_{\odot}$, which is consistent with the oxygen mass of $0.53 M_{\odot}$ estimated for the optimal model.

Baklanov et al. (2005) carry out the hydrodynamic study of SN 1999em in multi-group approximation, compare the calculated hydrodynamic model to both the observed UBVRI light curves and the photospheric velocity, and advocate the hydrodynamic model for the distance of $7.5 \mathrm{Mpc}$ with the pre-SN radius of $450 R_{\odot}$, the ejecta mass of $15 M_{\odot}$, and the explosion energy of $0.7 \times 10^{51} \mathrm{erg}$. However, the low metallicity of $Z=0.004$ for a red giant pre-SN and the uniform chemical composition with $X=0.7$ and $Z=0.004$ from the surface to its center are questionable. It is worth noting that their model for the distance of $12 \mathrm{Mpc}$ is not far from our optimal model in the basic parameters: the pre-SN radius of $1000 R_{\odot}$, the ejecta mass of $18 M_{\odot}$, and the explosion energy of $10^{51} \mathrm{erg}$.

The ejected envelope of $19 M_{\odot}$ and a neutron star of $1.58 M_{\odot}$ in the optimal model of SN $1999 \mathrm{em}$ correspond to a $20.58 M_{\odot}$ pre-SN star. Assuming an extremely low mass loss, we may now conclude that the lower mass limit of the progenitor of SN $1999 \mathrm{em}$ on the main sequence is nearly $21 M_{\odot}$. Heger et al. (2000) report the results of stellar evolution calculations of nonrotating main-sequence stars in the mass range of 10-25 $M_{\odot}$. It turns out that a main-sequence star of $25 M_{\odot}$ evolves to the preSN star of $18.72 M_{\odot}$ with the final helium core mass of $7.86 M_{\odot}$. A linear extrapolation for the pre-SN mass of $20.58 M_{\odot}$ yields the stellar mass of $28.9 M_{\odot}$ on the main sequence and the final helium core mass of $9.6 M_{\odot}$. Thus, the pre-SN of SN 1999em most likely developed from a main-sequence star in the mass range of 21-29 $M_{\odot}$.

Generally speaking, there is an opportunity to put constraints on the masses and the evolutionary states of the progenitors of core collapse SNe from direct observations. But it is a difficult task to identify the progenitors of SNe, and it is impossible to predict the time of explosion of a massive star precisely. 
For these reasons Smartt (2002) suggests estimating the initial masses of the progenitors by directly identifying SNe progenitors in the archive images of outburst sites taken prior to the explosions, by estimating the bolometric luminosity limits of progenitors as a function of stellar effective temperature, and by comparing them with stellar evolutionary tracks on the Hertzsprung-Russell diagram. Applying this method to SN 1999em, Leonard et al. (2003) have derived the upper mass limit for the progenitor of $20 \pm 5 M_{\odot}$ for the Cepheid distance to NGC 1637, and Smartt et al. (2003) obtained the upper limit of $15 M_{\odot}$ for the distance of $11 \mathrm{Mpc}$. Evidently, our estimate of the progenitor mass is in good agreement with the first upper mass limit.

It should be emphasized that the estimates of the bolometric luminosity for progenitors are hardly affected by the circumstellar dust, and a comparison with stellar evolutionary tracks is far from being unambiguous as the well-known case of SN 1987A demonstrated. There is growing observational evidence of the crucial role played by circumstellar dust in evaluating the bolometric luminosity of a progenitor star. For example, Barlow et al. (2005) show that a large fraction of the 5 mag of extinction within the host galaxy NGC 6946 toward SN 2002hh might be due to circumstellar gas dust, which might condense within a stellar wind from an earlier $M$ supergiant or luminous blue variable phase of the evolution of the progenitor star. And Massey et al. (2005) find that a significant fraction of red supergiants in Galactic OB associations and clusters show up to several magnitudes of excess visual extinction compared to OB stars in the same regions and argue that this is very likely due to circumstellar dust around the red supergiants. Note that the existence of circumstellar dust before an SN outburst does not contradict the lack of influence from dust on the photometric properties of an SN. Dust grains are most likely evaporated by the intense radiation flash during the shock breakout inside a sphere of radius of $\sim 0.1 \mathrm{pc}$ (Dwek 1983).

A good fit of the calculated bolometric light curve for the optimal model to that observed for SN 1999em is achieved with mutual mixing of the hydrogen-rich and helium-rich matter at the helium/hydrogen composition interface. The sharp boundary between the hydrogen-rich and helium-rich layers (Fig. 16a) at the edge of the helium core - a characteristic of the evolutionary models of pre-SNe - changes the light curve producing a distinct bump at the end of the plateau (Fig. 16b). Note that the same feature was obtained by Chieffi et al. (2003) in modelling the theoretical light curves of SNe IIP with the evolutionary models of pre-SNe. In addition to a deep hydrogen mixing in velocity space downward to $\approx 700 \mathrm{~km} \mathrm{~s}^{-1}$, the optimal model of SN $1999 \mathrm{em}$ is characterized by a weak ${ }^{56} \mathrm{Ni}$ mixing up to $\approx 660 \mathrm{~km} \mathrm{~s}^{-1}$ (Fig. 2 and Table 6).

The hydrodynamic model for SN 1987A is, in turn, characterized by a moderate ${ }^{56} \mathrm{Ni}$ mixing in velocity space up to $\sim 3000 \mathrm{~km} \mathrm{~s}^{-1}$ and a deep hydrogen mixing downward to $\sim 600 \mathrm{~km} \mathrm{~s}^{-1}$ (Utrobin 2005). Moreover, the observations of SN 1987A provide clear evidence of moderate mixing of the bulk of radioactive ${ }^{56} \mathrm{Ni}$ up to velocity $\sim 3000 \mathrm{~km} \mathrm{~s}^{-1}$ and deep hydrogen mixing down to $\sim 500 \mathrm{~km} \mathrm{~s}^{-1}$. For example, the [Ni II] $6.64 \mu \mathrm{m}$ profile at day 640 gives a velocity $v_{\mathrm{FWHM}}=$ $3100 \mathrm{~km} \mathrm{~s}^{-1}$ and its modelling results in a maximum velocity of $2600 \mathrm{~km} \mathrm{~s}^{-1}$ (Colgan et al. 1994). The fact that the H $\alpha$ profile on day 498 (Phillips et al. 1990) is not flat-topped implies that there is no large cavity free of hydrogen at the center of the envelope and that hydrogen is mixed downward to $\sim 500 \mathrm{~km} \mathrm{~s}^{-1}$.

Within the framework of the neutrino-driven explosion mechanism for core collapse SNe, Kifonidis et al. (2003, 2006) carried out two-dimensional simulations of explosion models with high-order mode perturbations and those with low-order ( $l=2$ and $l=1$ ) unstable modes induced by the initial global deformation of the shock. The low-mode explosion models exhibit final iron-group velocities of $\sim 3300 \mathrm{~km} \mathrm{~s}^{-1}$ and deep hydrogen mixing downward to a velocity of $\sim 500 \mathrm{~km} \mathrm{~s}^{-1}$, which are consistent with the observations of SN 1987A. The moderate ${ }^{56} \mathrm{Ni}$ mixing up to a velocity of $\sim 3300 \mathrm{~km} \mathrm{~s}^{-1}$ is the result of the larger initial maximum velocities of metal-rich clumps, compared to the high-mode models, which protect the fastest clumps from the strong interaction with the reverse shock that forms below the helium/hydrogen composition interface. The initial global deformation of the shock leads to the growth of the Richtmyer-Meshkov instability that in turn results in the strong inward mixing of hydrogen at the helium/hydrogen composition interface.

It is likely that the strongly anisotropic explosions are able to explain both weak and moderate ${ }^{56} \mathrm{Ni}$ mixing, depending on the pre-SN structure and the character of the interaction between metal-rich clumps and the reverse shock. Thus, the weak ${ }^{56} \mathrm{Ni}$ mixing and the deep hydrogen mixing, which are the characteristic features of the optimal model for SN 1999em, cannot be excluded within the framework of the neutrino-driven explosion mechanism (Kifonidis 2006). These promising capabilities make our interpretation of the SN 1999em event more physically grounded and confirm the suggestion of Chieffi et al. (2003) that in reality the inner layers of the expelled envelope are strongly mixed during the explosion.

Based on the hydrodynamic models of SNe IIP, Litvinova \& Nadyozhin $(1983,1985)$ constructed simple approximate formulae for evaluating the initial radius, the ejecta mass, and the explosion energy - three basic physical parameters - from the observed properties of individual SN IIP. Using these approximate formulae for SN 1999em and assuming the distance to the host galaxy to be $11.08 \mathrm{Mpc}$, Nadyozhin (2003) derived the initial radius of $414 R_{\odot}$, the ejecta mass of $15.0 M_{\odot}$, and the explosion energy of $0.68 \times 10^{51} \mathrm{erg}$. The difference in the ejecta mass and the explosion energy, compared to those of the optimal model, results mainly from neglecting the influence of radioactive ${ }^{56} \mathrm{Ni}$ on the light curve and using the approximation of equilibrium radiative diffusion in their hydrodynamic models.

We go one step further to construct the approximate formulae (2-4), which include the influence of radioactive ${ }^{56} \mathrm{Ni}$ on the light curve and are based on the hydrodynamic models computed in terms of radiation hydrodynamics in the one-group approximation with non-LTE effects in the average opacities and the thermal emissivity, with nonthermal ionization, and with the contribution of lines to opacity. The resulting approximate formulae, obtained in space of the physical parameters, are valid only for the vicinity of the optimal model because of the sensitivity of the bolometric light curve to the pre-SN structure and to its chemical composition. To construct the global, approximate relations covering a wider range of the parameters, we have to use a set of hydrodynamic models computed for many real SNe IIP. The optimal model for SN 1999em is the first element in this set.

\section{Conclusions}

We have presented a comprehensive study of a normal type IIP SN $1999 \mathrm{em}$. Our main aim in this paper was to show the absolute necessity of the simultaneous interpretation of both the photometric and spectroscopic observations. To do this, we calculated the hydrodynamic models of SN 1999em complemented by the atmosphere models with the time-dependent kinetics and 
energy balance. We focused mainly on the optimal hydrodynamic model of SN 1999em and subsequent comparison to a peculiar type IIP SN 1987A. Our results can be summarized in the following conclusions.

- The bolometric light curve of SN 1999em and its spectral evolution of the $\mathrm{H} \alpha$ line are consistent with a radius of the pre-SN of $500 \pm 200 R_{\odot}$, a mass of the ejected envelope of $19.0 \pm 1.2 M_{\odot}$, an explosion energy of $(1.3 \pm 0.1) \times 10^{51} \mathrm{erg}$, and a mass of radioactive ${ }^{56} \mathrm{Ni}$ of $0.036 \pm 0.009 M_{\odot}$, the bulk of which is confined to layers ejected with velocities less than $\approx 660 \mathrm{~km} \mathrm{~s}^{-1}$. The optimal hydrodynamic model with the helium core mass in the range of 5.6-8.1 $M_{\odot}$ matches the observed bolometric light curve.

- The adequate hydrodynamic and atmosphere models of SN 1999em distinguish between the short distance of $7.85 \mathrm{Mpc}$, the average value of the EPM distance estimates to the host galaxy, and the Cepheid distance of $11.7 \mathrm{Mpc}$. They are inconsistent with the short distance of $7.85 \mathrm{Mpc}$, which should be discarded.

- It is shown that the hydrogen recombination in the atmosphere of a normal type IIP SN $1999 \mathrm{em}$, as well as most likely other SNe IIP, at the photospheric epoch is essentially a time-dependent phenomenon in accordance with the analysis of Utrobin \& Chugai $(2002,2005)$.

- This study reveals the time development of a normal SN IIP with the following stages: a shock breakout, an adiabatic cooling phase, a phase of cooling and recombination wave, a phase of radiative diffusion cooling, an exhaustion of radiation energy, a plateau tail, and a radioactive tail.

- The plateau tail of the theoretical bolometric light curve coinciding in time with the second plateau observed in SN 1999em (Elmhamdi et al. 2003) runs slightly above a line of the radioactive tail in contrast to the latter. It seems that the real bolometric luminosity of SN $1999 \mathrm{em}$ is greater than the "UBVRI' luminosity during the second plateau. The duration of the plateau tail, as well as the second plateau, is correlated with the amount of ejected ${ }^{56} \mathrm{Ni}$.

- The bolometric light curve depends weakly on the chemical composition in the outer layers of the ejecta beyond the helium core, while hydrogen is abundant there and controls the opacity of matter. A mutual mixing of hydrogen-rich and helium-rich matter in the inner layers of the ejecta guarantees a good fit of the calculated bolometric light curve to what is observed. Note that the sharp boundary between the hydrogen-rich and helium-rich layers at the edge of the helium core - a characteristic of the evolutionary models of pre-SNe - changes the light curve producing an unobserved bump at the end of the plateau.

- A contribution of numerous metal lines to opacity plays a fundamental role in reproducing the observed light curve of SN $1999 \mathrm{em}$. The decrease in opacity due to disregarding the line contribution speeds up the radiation diffusion and causes the bolometric luminosity to increase significantly compared to what is observed during nearly the whole outburst and, as a consequence, causes the characteristic duration of the light curve to shorten by about 10 days.

- The comparison of a normal type IIP SN $1999 \mathrm{em}$ with a peculiar type IIP SN 1987A reveals two very important results for SN theory. First, the masses of helium cores in the pre-SNe of SN 1999em and SN 1987A are close enough to suppose that in the final stages of stellar evolution nearly the same iron cores form within the pre-SNe. This fact and roughly the same explosion energies of SN 1999em and
SN 1987A together imply a unique explosion mechanism for these core collapse SNe. Second, the optimal model for $\mathrm{SN} 1999 \mathrm{em}$ is characterized by a weaker ${ }^{56} \mathrm{Ni}$ mixing in velocity space up to $\approx 660 \mathrm{~km} \mathrm{~s}^{-1}$ compared to a moderate ${ }^{56} \mathrm{Ni}$ mixing up to $\sim 3000 \mathrm{~km} \mathrm{~s}^{-1}$ in the case of SN 1987A, hydrogen being mixed deeply downward to $\approx 700 \mathrm{~km} \mathrm{~s}^{-1}$ and $\sim 600 \mathrm{~km} \mathrm{~s}^{-1}$.

- A significant excess of the gamma-ray luminosity over the bolometric one, shown by the optimal model of SN 1987A at the radioactive tail, is of great interest and should be taken into account in measuring the ${ }^{56} \mathrm{Ni}$ amount from the observed bolometric luminosity at this stage when the SN envelope still remains optically thick for gamma-rays. The higher the rate of work done by radiation pressure in an SN envelope, the larger an excess of the gamma-ray luminosity over the bolometric one at the radioactive tail.

- It is shown that the approximation of homologous expansion may be used in the EPM and SEAM for determining the distances to normal SNe IIP starting from nearly day 3 after the SN explosion.

- Based on the hydrodynamic models in the vicinity of the optimal model of SN $1999 \mathrm{em}$, we derive the approximate relationships between the basic physical and observed parameters. The basic physical parameters are the pre-SN radius, the ejecta mass, the explosion energy, and the total ${ }^{56} \mathrm{Ni}$ mass. The observed properties of SNe IIP are described by the three parameters: the characteristic duration of the light curve, the bolometric luminosity and the photospheric velocity in the middle of the plateau. To cover a wider range of the parameters, beyond the vicinity of the optimal model, we have to construct a global approximation that should be based on a set of hydrodynamic models of many real SNe IIP.

Finally, we emphasize that the simultaneous analysis of the photometric and spectroscopic observations is in fact crucial for correctly interpreting core collapse $\mathrm{SNe}$ and express the hope that the obtained basic parameters and properties of SN $1999 \mathrm{em}$ determine its evolutionary state and will be a reliable reference for the proper mechanism of SN explosions.

Acknowledgements. The author is grateful to Wolfgang Hillebrandt and Ewald Müller for hospitality during his stay at the MPA. The author would also like to thank Nikolai Chugai, Konstantinos Kifonidis, and Dmitrij Nadyozhin for many discussions, and the referee David Branch for helpful comments. This work was supported in part by the Russian Foundation for Fundamental Research (04-01-17255).

\section{References}

Arnett, W. D. 1979, ApJ, 230, L37

Arnett, W. D. 1987, ApJ, 319, 136

Baklanov, P. V., Blinnikov, S. I., \& Pavlyuk, N. N. 2005, Astron. Lett., 31, 429

Barlow, M. J., Sugerman, B. E. K., Fabbri, J., et al. 2005, ApJ, 627, L113

Baron, E., Branch, D., Hauschildt, P. H., et al. 2000, ApJ, 545, 444

Baron, E., Nugent, P. E., Branch, D., \& Hauschildt, P. H. 2004, ApJ, 616, L91

Basko, M. 1994, ApJ, 425, 264

Chieffi, A., Domínguez, I., Höflich, P., Limongi, M., \& Straniero, O. 2003, MNRAS, 345, 111

Colgan, S. W. J., Haas, M. R., Erickson, E. F., Lord, S. D., \& Hollenbach, D. J. 1994, ApJ, 427, 874

Dessart, L., \& Hillier, D. J. 2006, A\&A, 447, 691

Dufour, R. J. 1984, in Structure and Evolution of the Magellanic Clouds, ed. S. van den Bergh, \& K. S. de Boer (Dordrecht: Reidel), IAU Symp., 108, 353 Dwek, E. 1983, ApJ, 274, 175

Elmhamdi, A., Danziger, I. J., Chugai, N., et al. 2003, MNRAS, 338, 939

Falk, S. W., \& Arnett, W. D. 1977, ApJS, 33, 515

Grassberg, E. K., \& Nadyozhin, D. K. 1976, Ap\&SS, 44, 409

Grassberg, E. K., Imshennik, V. S., \& Nadyozhin, D. K. 1971, Ap\&SS, 10, 28 
Grassberg, E. K., Imshennik, V. S., Nadyozhin, D. K., \& Utrobin, V. P. 1987, Astron. Lett., 13, 227

Grevesse, N., \& Sauval, A. J. 1998, Space Sci. Rev., 85, 161

Hamuy, M. 2003, ApJ, 582, 905

Hamuy, M., Pinto, P. A., Maza, J., et al. 2001, ApJ, 558, 615

Heger, A., Langer, N., \& Woosley, S. E. 2000, ApJ, 528, 368

Hillebrandt, W., Höflich, P., Truran, J. W., \& Weiss, A. 1987, Nature, 327, 597

Imshennik, V. S., \& Nadyozhin, D. K. 1989, Astrophys. Space Phys. Rev., 8, 1

Kifonidis, K. 2006, private communication

Kifonidis, K., Plewa, T., Scheck, L., Janka, H.-Th., \& Müller, E. 2003, A\&A, 408, 621

Kifonidis, K., Plewa, T., Scheck, L., Janka, H.-Th., \& Müller, E. 2006, A\&A, 453, 661

Kirshner, R. P., \& Kwan, J. 1974, ApJ, 193, 27

Leonard, D. C., Filippenko, A. V., Ardila, D. R., \& Brotherton, M. S. 2001, ApJ, 553,861

Leonard, D. C., Filippenko, A. V., Gates, E. L., et al. 2002, PASP, 114, 35

Leonard, D. C., Kanbur, S. M., Ngeow, C. C., \& Tanvir, N. R. 2003, ApJ, 594, 247
Li, W. D. 1999, IAU Circ., 7294

Litvinova, I. Yu., \& Nadyozhin, D. K. 1983, Ap\&SS, 89, 89

Litvinova, I. Yu., \& Nadyozhin, D. K. 1985, Sov. Astron. Lett., 11, 145

Massey, P., Plez, B., Levesque, E. M., et al. 2005, ApJ, 634, 1286

Mihalas, D. 1978, Stellar Atmospheres (San-Francisco: Freeman)

Nadyozhin, D. K. 2003, MNRAS, 346, 97

Phillips, M. M., Hamuy, M., Heathcote, S. R., Suntzeff, N. B., \& Kirhakos, S. 1990, AJ, 99, 1133

Pooley, D., Lewin, W. H. G., Fox, D. W., et al. 2002, ApJ, 572, 932

Powell, M. J. D. 1964, Comput. J., 7, 155

Smartt, S. J. 2002, Ap\&SS, 281, 187

Smartt, S. J., Maund, J. R., Gilmore, G. F., et al. 2003, MNRAS, 343, 735

Sobolev, V. V. 1980, Afz, 16, 695

Utrobin, V. P. 1989, Astron. Lett., 15, 42

Utrobin, V. P. 2004, Astron. Lett., 30, 293

Utrobin, V. P. 2005, Astron. Lett., 31, 806

Utrobin, V. P., \& Chugai, N. N. 2002, Astron. Lett., 28, 386

Utrobin, V. P., \& Chugai, N. N. 2005, A\&A, 441, 271

Woosley, S. E. 1988, ApJ, 330, 218 